\title{
Astrologues et devins du Koryŏ (918-1392) : une analyse de l'histoire officielle
}

Astrologers and Divination Specialists of the Koryŏ (918-1392): an Analysis of the official History

\section{Yannick Bruneton}

\section{OpenEdition Journals}

Édition électronique

URL : http://journals.openedition.org/extremeorient/278

DOI : 10.4000/extremeorient.278

ISSN : 2108-7105

\section{Éditeur}

Presses universitaires de Vincennes

\section{Édition imprimée}

Date de publication : 1 mai 2013

Pagination : 45-81

ISBN : 978-2-84292-367-9

ISSN : 0754-5010

Référence électronique

Yannick Bruneton, «Astrologues et devins du Koryŏ (918-1392) : une analyse de l'histoire officielle », Extrême-Orient Extrême-Occident [En ligne], 35 | 2013, mis en ligne le 01 mai 2016, consulté le 20 avril 2019. URL : http://journals.openedition.org/extremeorient/278 ; DOI : 10.4000/extremeorient.278 


\title{
Astrologues et devins du Koryŏ (918-1392) : une analyse de l'histoire officielle
}

\author{
Yannick Bruneton
}

\section{Introduction}

De multiples indices de la culture écrite du Koryŏ (dynastie des Wang, 918-1392) prouvent que les pratiques divinatoires, déclinées selon leurs différents domaines d'application, étaient largement répandues dans toute la société médiévale de la Corée. En témoignent des regards extérieurs à la péninsule coréenne comme celui de Yelü Chun (1062-1122) des Liao (9071125), compilateur du traité de divination astrale du Xingming zonggua (984 ?), prétendant être le dépositaire des savoirs d'un «maître du pays» guoshi du Koryŏ ${ }^{1}$, ou bien de Xu Jing (1091-1127) des Song du Nord (960-1126) envoyé à la cour de Kaegyŏng (actuel Kaesŏng) au début du XII ${ }^{\mathrm{e}}$ siècle. Outre le fait que l'intervention des devins et astrologues semble avoir été requise aux moments d'étapes charnières de l'existence, les praticiens des arts divinatoires auraient été issus de statuts sociaux situés à divers degrés de l'échelle sociale. Dans ces conditions, la connaissance des arts divinatoires du Koryŏ, des théories qui les sous-tendent, des conditions et des enjeux de leur pratique, de l'identité et des motivations de leurs praticiens et de leurs destinataires, s'avère constituer une donnée de premier ordre pour comprendre la société de l'époque. De plus, parce qu'en ce domaine les techniques les plus formalisées proviennent de l'État impérial chinois, l'analyse des sources et des pratiques divinatoires de la Corée médiévale présente également l'intérêt de nous renseigner sur la nature des relations entre royaume coréen et empire en termes de circulation des savoirs.

1. 星命總括. Litt. «Somme générale sur la destinée [de naissance] par les astres». Selon la préface de l'ouvrage, l'auteur aurait été membre d'une légation envoyée à la cour du Koryŏ en 984, mais l'information n'est pas corroborée par les sources coréennes. Voir aussi le Chibong ryusŏl 芝峰類說: 18 (技藝部: 方術, 星曜書). 
Cependant, l'état sinistré de la production écrite de la période rend délicate la description des pratiques ainsi que la connaissance des détenteurs des techniques. Le caractère limité et dispersé des données explique sans doute la rareté des études menées jusqu'à présent sur les astrologues et devins du Koryŏ en Corée du $\mathrm{Sud}^{2}$, le pays le plus avancé dans les recherches historiques sur la période. Par commodité, la recherche s'est focalisée sur l'analyse des «astrologues» ilgwan, le terme le plus utilisé pour désigner les devins d'État dans l'histoire dynastique, le Koryŏsa (Histoire du Koryŏ, 1451) .

En effet, l'histoire officielle du Koryŏ constitue la principale source d'informations dont nous disposons aujourd'hui à propos des arts divinatoires de l'époque. Toutefois, force est de constater qu'il y existe peu de lieux précis de description de l'activité et d'expression directe des acteurs de la divination d'État. À cette limite fondamentale, il faut ajouter que les mentions, qui les concernent, comportent une valeur idéologique du point de vue de l'historiographie: en comprendre la nature est essentiel pour analyser les sources officielles de la Corée. En effet, dans le cadre de la pensée confucianiste orthodoxe, il semble que les pratiques divinatoires aient été conçues de manière hiérarchisée avec une tolérance proportionnelle à leur degré de conformité à la doctrine. Dans l'histoire officielle du Koryŏ, la mention sélective de la divination agit comme un révélateur de la moralité de la politique des souverains: les calamités étant comprises comme des «blâmes du Ciel» ch'ŏn kyŏn ${ }^{4}$. Ainsi, les devins étaient utiles dès lors que leur tâche consistait à les révéler, mais les remèdes qu'ils préconisaient, eux, étaient souvent implicitement critiqués.

La présente contribution cherche à mettre en évidence le fonctionnement de la divination d'État du Koryŏ à partir d'une analyse des acteurs de la divination telle qu'elle est rapportée dans le Koryŏsa, sur la base des questions suivantes: de quels dispositifs institutionnels étaient-ils issus, comment étaient-ils formés,

2. L'article de fond remarquable de Kim Ch'anghyŏn fait exception ( $c f$. Bibliographie). J'ai utilisé ici largement les résultats de son travail pour ce qui concerne l'analyse des institutions et les carrières des devins d'État. D'une manière générale, la présente contribution reflète l'état de la recherche en Corée du Sud.

3. 高麗史. Histoire dynastique en «annales et biographies» (紀傳體), abrégée en plan chronologique (編年體) dans la version du Koryŏsa chŏryo 高麗史節要 (publiée en 1452), qui n'en est toutefois pas l'exact résumé (la consultation des deux ouvrages est indispensable).

4. Les textes du Koryŏ qui traitent de cette thèse fondamentale sont par exemple: la discussion de Im Wan (林完,?-?, XII ${ }^{\mathrm{e}}$ s.; chaei sangs TMS : 52), un rapport de Yi Kyubo (1168-1241): Chisihwang pulbun chuyŏk non 秦始 皇不焚周易論; TMS : 99; le rapport de Kwŏn Kyŏngjung (KRS : 101,12b-21a). KRS : 2,2b.26a; 7,40a; 8,10;20,36a; 29,38a; 55,5a; 117,28b; 122,7b; 133,25a. Le terme ch'ŏn'gyŏn (天譴) est mentionné une douzaine de fois dans le KRS. 
recrutés, et quelles étaient leurs activités? À quels traités se référaient-ils et quelles sources produisirent-ils? Comment étaient-ils organisés ? Sous quelles modalités leurs savoirs furent-ils transmis ? Finalement, comment caractériser les devins et la culture divinatoire spécifique du Koryŏ en dépit des limites des sources?

\section{Le dispositif institutionnel et la littérature spécialisée}

\section{Les bureaux spécialisés}

Comprendre le fonctionnement de la divination sous la dynastie des Wang requiert la connaissance des institutions qui servirent de cadre à ses pratiques. L'administration en charge de l'astrologie et de la divination servit en effet de référence aux devins officiels et privés.

\section{Dans la capitale principale}

Dans le «traité sur l'Administration» du Koryŏsa, sous l'appellation de Sŏun'gwan (litt. «Office de la rédaction de l'observation des nuages $»^{5}$ ), est relaté, selon l'usage historiographique chinois, l'historique du bureau de la Divination. L'exposé préliminaire des fonctions ${ }^{6}$ qui y étaient théoriquement assurées est incomplet: à l'astronomie ch'ŏnmun, aux lots calendériques yŏksu, aux prévisions climatiques $c h^{\prime} \breve{u k} k^{\prime} h u$ et au service de la clepsydre kangnu, il faut ajouter l'observation des phénomènes naturels extraordinaires chaesang ${ }^{7}$, les pronostics par tirage poks $\breve{o}^{8}$ et la géomancie $c h i r i^{9}$, la prévision des éclipses et le choix des dates fastes sŏnt'aek, l'indication de l'heure ${ }^{10}$. La date

5. 書雲觀. Appellation de l'époque des Yuan, utilisée au Koryŏ à partir de 1308 (KRS : 76,45a).

6. À l'époque des Han, Liu Xin (劉歆, -50 ?; -23), astrologue et historiographe des Xin, définit six principaux domaines d'application des écoles du yin et du yang: «astronomie, almanachs, Cinq agents, tiges d'achillée et [carapaces de] tortues, pronostics divers, école des formes» (天文, 歷譜, 五行, 著龜, 雜占, 形法; Kim 1992: 97). Kim 1992: 98.

7. 災祥. CWS : 1392.7 .28 .

8. 卜筮. 4 occurrences dans le KRS.

9. 地理. Dans le KRS, l'autre terme utilisé pour désigner la géomancie est p’ungsu (風 水).

10. Cf.Ch’ŭngbo Munhŏn pigo 增補文獻備考: 223, 職官考 10, 觀象監(本朝 太祖元年 因麗制 置書雲觀 掌天文 地理 曆數 測候 漏刻 等事). CWS : 1392.7 .18 (定文武 百官之制... 書雲觀 掌天文 災祥 曆日 推擇 等事). Kim recense sept principales attributions des devins fonctionnaires du Koryŏ : 1) l'observation des phénomènes célestes (天文) et les méthodes d'éradication des irrégularités;2) la rédaction de 
de fondation du Sŏun'gwan est imprécise («début du pays»), mais l'office aurait été dès l'origine divisé en deux bureaux ${ }^{11}$, dont l'un était spécialisé dans l'astronomie ${ }^{12}$, l'autre dans les pronostics (et la géomancie) ${ }^{13}$, avec un nombre indéterminé de membres. Il faut attendre le règne de Munjong (r. 1046-1083) ${ }^{14}$ pour qu'une nomenclature de référence soit fixée: deux ensembles de onze et quinze fonctionnaires rattachés respectivement au sach'ŏndae (litt. «terrasse des Affaires célestes ») et au t'aesaguk (litt. «bureau du Grand Historiographe ») ${ }^{15}$.

calendriers (撰曆) servant à la prédiction des phénomènes remarquables comme les éclipses, et aussi à la prévention des calamités ; 3) l'harmonie des Cinq agents à l'origine des phénomènes terrestres et climatiques : relevé des épisodes et recommandations des méthodes pour les éliminer; 4) pronostics par tirage sur le caractère faste d'une affaire importante (déplacement du roi, transfert de la capitale, sortie de l'armée régulière); 5) choix des dates fastes (funérailles, réception d'un décret, rites, sortie de l'armée); 6) la clepsydre (horloge à eau; parfois ambulante) : annonce des heures pour les grandes cérémonies (dans le temple des ancêtres de la dynastie, les fêtes bouddhiques annuelles des Lanternes et des Huit commandements); 7) la géomancie (choix des sites fastes pour les funérailles, la construction de palais, d'édifices bouddhiques). En raison de telles attributions, les devins participaient aux escortes du roi en déplacement (扈從), accompagnaient les armées (從軍), assuraient des tours de veille (直宿), intervenaient dans les procédures de rites sacrificiels (祭儀): installation des tablettes des esprits notamment (Kim 1992: 100-106).

11. Pour Kim Ch'anghyŏn, ce fut d'abord le sach'ŏndae qui fut mis en place sous T'aejo. La première apparition du terme t'aesaguk dans les sources daterait de 981, et de 993 pour t'aebokkam (Kim 1992: 85).

12. Le t'aebokkam aurait été spécialisé dans les pronostics par tirage poksŏ. Dans son Répertoire Historique de l'administration coréenne, Maurice Courant associe le t'aebokkam 太卜監 $\left(\mathrm{n}^{\circ}\right.$ 600) au bureau de la Divination et le t'aesaguk 太史局 au bureau de l'Astrologie ( $\left.n^{\circ} 602\right)$.

13. Kim 1992: 92. Pour Kim Haeyŏng, la division en deux bureaux s'explique pour des raisons fonctionnelles. Cependant, comme le montre bien Kim: 1) les membres des deux entités se partageaient des tâches similaires (notamment en ce qui concerne la géomancie); 2) les carrières s'effectuaient par des passages d'un bureau à l'autre; 3 ) ils sont qualifiés de ilgwan 日官 astrologues (ou ilcha 日者) de manière quasi indifférenciée par les historiographes (Kim 1992: 94-95). La tendance à une véritable spécialisation des compétences divinatoires par domaine d'application n'apparaît qu'à la fin de la période avec la création des dix écoles (KRS : 77,29a-b).

14. Munjong accomplit une refonte de l'administration en 1076. T'aejo, le fondateur, et Munjong constituent selon les historiographes, les deux règnes de référence pour les institutions de la dynastie.

15. La nomenclature est très proche de celle des Tang dont elle s'inspire (Kim 1992: 87), avec quelques différences, notamment dans les appellations de charges (Kim 1992: 88). D’un point de vue économique, Munjong porta les classes de rétributions foncières 
Une telle configuration est cependant tout à fait insuffisante à rendre compte des activités en lien avec la divination à l'échelle du territoire.

En dehors de changements d'appellations, la principale réforme qu'aurait connue l'institution fut la fusion des deux bureaux ${ }^{16}$ par le roi Ch'ungsŏn (r. 1308-1313), suivie d'une réduction du nombre des fonctionnaires ${ }^{17}$. Kongmin (r. 1351-1374), en 1356, rétablit l'ancienne nomenclature de Munjong, puis la révisa temporairement ${ }^{18}$. Le dispositif ainsi décrit reprend les appellations chinoises des Tang, des Song et des Yuan, révélant l'influence de l'administration impériale comme modèle, à la différence des institutions du Silla (dates officielles: $-57,935)$. La rubrique omet de plus de signaler les relations entre les deux bureaux ${ }^{19}$, ainsi que leur rattachement (au ministère des Rites ou au cabinet des Livres pisŏsŏng) ${ }^{20}$. En 1389, le Sŏun'gwan devint le lieu d'accueil des écoles de géomancie p'ungsuhak et du yin-yang ümyanghak ${ }^{21}$, indiquant par là une tendance à la spécialisation des connaissances divinatoires.

Un des aspects de la divination d'État passé sous silence est la description du système de relais existant entre l'administration centrale et les bureaux locaux pour le relevé des phénomènes naturels. Nous ignorons les modalités de production et de circulation de l'information entre la capitale et la province sans lesquels la consignation des 3124 notations du traité des Cinq agents aurait été impossible. Il est vraisemblable que le bureau d'Astrologie comportait des annexes provinciales dont les membres effectuaient des relevés climatiques et inspectaient les lieux symbolisant le pouvoir dynastique. Un inventaire manuscrit de 1230 rapportant l'ensemble des possessions du monastère de Susŏn ${ }^{22}$, signé

rétribuant les charges des devins fonctionnaires au même niveau que celles des autres administrations à échelon équivalent (Kim 1992: 121).

16. La tendance à la fusion s'observe également dans les institutions équivalentes des Tang (Kim 1992: 88).

17. Pour le détail, $c f$. Kim 1992: 90.

18. Pour l'historien Min Hyŏn'gu, les quatre vagues successives de réformes concernaient l'ensemble de l'administration et s'expliquent principalement par les fluctuations en matière de relations internationales (Kim 1992: 91, note 61). Le bureau fut réformé sous la dynastie suivante à partir de 1392 (CWS : 1392.7.28).

19. Pour Kim, le t'aesaguk était rattaché au sach'ŏndae, comme le révèle la hiérarchie comparée de leur nomenclature respective (Kim 1992: 95). Il faut ajouter un système de cumul des charges au sommet de la hiérarchie. Dans le manuscrit de 1230 ( $c f$. infra p. 4), la signature des fonctionnaires du t'aesaguk est accompagnée de l'apposition du sceau du sach'ŏndae (Kim 1992: 96).

20. Kim 1992: 95. Aucun élément ne précise leur rattachement.

21. KRS : 77,29a-b (置... 風水陰陽等學于書雲觀).

22. 修禪社. Actuel monastère de Songgwang (松廣寺; Sunch’ŏn, prov. Chŏlla du Sud). Hŏ 1994 : 269-271. 
de la main du sasin (litt. «préposé aux heures ») Yun Ch'ang (?- ?), et du saryŏk (litt. «préposé au calendrier») Sim Chun (?- ?), deux charges de devins de $9^{\text {e }}$ (dernier) échelon, suggère l'existence de tels préposés dans les provinces. Nous savons en revanche qu'il existait des institutions autonomes dans une des capitales secondaires du territoire.

\section{L'école de divination de la capitale de l'Ouest}

Établie grande préfecture de défense dès 918, puis élevée au rang de capitale secondaire (capitale de l'Ouest) ${ }^{23}$, P'yŏngyang fut l'objet de l'attention de T'aejo Wang Kŏn (r. 918-943), le fondateur de la dynastie. Outre ses multiples déplacements dans la cité, il la dota d'un système de fortifications, la repeupla et en fit un centre urbain, démographique, et surtout militaire. La cité de l'Ouest constituait ainsi le poste avancé en vue de la reconquête des anciens territoires du Koguryŏ, dont la filiation avec le Koryŏ était revendiqué par le choix du nom de pays ${ }^{24}$. Les historiographes laissent entendre que T'aejo conçut le dessein de faire de P'yŏngyang la capitale principale, projet qu'il aurait abandonné en 932 en raison de la présence de signes jugés défavorables par les devins d'État ${ }^{25}$. La cité était néanmoins considérée comme un haut lieu de mémoire de l'histoire de la péninsule, un site fondateur du point de vue de la civilisation coréenne, justifiant des cultes nombreux et anciens ${ }^{26}$. À partir de 922, puis en 990 et en 1116, la capitale de l'Ouest ne cessa de s'enrichir d'un arsenal d'institutions séparées lui conférant autonomie vis-à-vis de la capitale principale, notamment dans les domaines de la défense, de la diplomatie et de la formation des lettrés fonctionnaires. Le statut ainsi acquis au XII ${ }^{\mathrm{e}}$ siècle était sans équivalent. Les mesures prises par Yejong (r. 1105-1122) aboutirent à la mise en place d'une annexe du bureau d'Astrologie, remplaçant le bureau de la Clepsydre ${ }^{27}$, avec un nombre de membres sans quota, dirigé par un haut fonctionnaire au titre de charge cumulée. Une telle promotion eut des conséquences importantes puisqu'elle permit l'émergence de devins, qui revendiquèrent, une douzaine d'années plus tard, le transfert de la capitale à P'yŏngyang ${ }^{28}$.

23. KRS : 77,36b. 西京 Sŏgyŏng.

24. Dans les sources chinoises, le Koguryŏ 高句麗 est souvent abrégé en Koryŏ 高麗. Voir aussi KRS : 94,4b.

25. KRS : $2,2 b$.

26. KRS : 58,31a (cultes à Jizi, Kija 箕子 et à Tongmyŏng 東明王, fondateur du Koguryŏ, et autres).

27. 漏刻院.

28. Cf. infra p. 67. 


\section{La formation, le recrutement et la carrière}

Dans la capitale, après l'instauration du concours de recrutement des fonctionnaires (958) inspiré du modèle des Tang, les aspirants devins étaient censés être formés au sein des sections spécialisées et subalternes chap $\breve{p} p$ dites de divination pok $\breve{o p}$ et de géomancie chiri $\breve{o p}{ }^{29}$. Malheureusement, à la différence d'autres sections, nous n'en connaissons pas les lauréats ${ }^{30}$. À partir de 1136, fut établie la liste des ouvrages de référence (pour la section de géomancie seulement) servant à tester les connaissances théoriques des futurs devins fonctionnaires ${ }^{31}$. Dans l'administration, le rôle des devins d'État était purement consultatif: in fine c'étaient les conseillers d'État (sinon le roi) qui décidaient. Toutefois, par leur relative liberté de parole (pour dénoncer indirectement les carences politiques) ${ }^{32}$, leur capacité de persuasion, leur implication dans les processus de décision, ils jouaient un rôle décisif dans la vie politique. Autrement dit, aucune action politique un peu ambitieuse ne pouvait se réaliser sans leur collaboration.

Du point de vue économique, les devins, à échelon égal, recevaient un traitement inférieur en «appointements et commis » à celui de leurs homologues civils et militaires, en tant que techniciens issus des matières subalternes du concours $^{33}$. Il semble que le rattachement à la section de géoscopie, au moins temporaire, rendait possible la rétribution d'experts indépendants. On trouve ainsi mention de rangs monastiques concernés par ce type de disposition ${ }^{34}$, révélant la reconnaissance de compétences de religieux dans le domaine. De fait, dans un système où la compétence primait ${ }^{35}$, il existait des modalités souples de recrutement des devins, autres que la formation par les sections spécialisées ${ }^{36}$.

29. 雜業: 卜業, 地理業. KRS : 73,1b.

30. Les historiographes ne consignent que les lauréats de la section de rédaction chesurŏp 製述業 ou bien les «protégés de l'État» ŭmsŏ 蔭敍, exemptés de concours.

31. Nous connaissons le déroulement des épreuves (KRS : 73,8a-b; Bruneton 2002: 298-301)

32. Kim 1994: 110-111.

33. À partir de 1136, les épreuves du concours furent assurées par les bureaux spécialisés (en l'occurrence le bureau d'Astrologie). Kim 1992: 127-128. Il existait le système de t'alma 脫麻 permettant à des candidats ayant étudié plus de dix ans d'entrer dans la carrière. Dans la capitale de l'Ouest, les candidats ayant étudié plus de vingt ans et en ayant plus de cinquante entraient dans la carrière (KRS: 74,6b).

34. KRS : 78,13a. Il semble que les milieux monastiques recouraient à des moines géomanciens locaux dans les procédures de construction des édifices bouddhiques. $C f$. Нŏ 1988 : 66.72 .261 .

35. Kim 1992: 140 .

36. Kim 1992: 132. Certains lettrés et membres de la famille royale (comme Wang Uk; KRS : 90,3a) excellaient dans la connaissance des techniques divinatoires. 
Toute personne jugée apte pouvait être recrutée pour les besoins de l'État dans des structures souples et collégiales de type «conseils temporaires » $\operatorname{togam}^{37}$. Ces cellules rendaient ainsi admissible la collaboration de séculiers et de religieux dans l'administration du Koryŏ, inimaginable dans le cadre plus rigide de l'État impérial.

Kim Ch'anghyŏn, qui a analysé les carrières de devins, conclut qu'il fallait une trentaine d'années pour qu'un fonctionnaire ayant débuté son parcours au bas de l'échelle parvienne à une charge de direction du bureau de la Divination. Il distingue aussi les devins ayant fait toute leur carrière dans le bureau (ou avec des passages entre le bureau de la Divination et celui d'Astrologie) des hauts fonctionnaires qui en occupèrent la direction en cumul. Le cumul des charges permit à certains d'intégrer une autre administration. Ainsi, en certaines circonstances (reconnaissance du mérite envers l'État), des devins fonctionnaires d'origine furent nommés conseillers d'État ${ }^{38}$. Du point de vue de l'origine sociale, les devins étaient principalement issus des clercs de province hyangni ou des ordres civil et militaire subalternes. Après la période de prise de pouvoir par les militaires (1170-1270), on constate des cas plus nombreux de nomination de militaires à des charges de divination: 1'art militaire de l'époque recourant fréquemment aux pronostics divinatoires ${ }^{39}$. À la fin de la période, le passage par un poste de devin aurait constitué pour les clans des chefs de localités une voie de transition dans un parcours visant une haute charge civile ${ }^{40}$.

En plus de reconstituer les trajectoires des devins au sein de l'administration, il est nécessaire de saisir la nature de leurs activités: pour cela, analyser les traités administratifs du Koryŏsa s'avère indispensable.

\section{Les traités astronomiques et divinatoires du Koryŏsa}

Dans l'histoire dynastique, trois mémoires administratifs reflètent les attributions concrètes des astrologues et devins officiels : le traité d'Astronomie, celui de Calendérologie et celui des Cinq agents ${ }^{41}$.

37. 都監. Bruneton $2002: 821$.

38. Kim 1992: 139.

39. 墽吏. Kim 1992: 144.

40. Kim 1992: 150 .

41. Ces traités ont été récemment retraduits (première traduction aux éditions de l'Université Yonsei) et minutieusement annotés par Kim Ilgwŏn en 2011 ( $c f$. Bibliographie). 


\section{Le traité d'Astronomie ch'ŏnmun chi}

Le traité d'Astronomie précède celui des calendriers et des Cinq agents ${ }^{42}$, au début de la Section des mémoires administratifs chi. On y décèle l'influence du Yuanshi dans la structure comme dans le contenu ${ }^{43}$. Deux grandes parties sont dédiées respectivement aux «éclipses de soleil» ilsik (peu nombreuses); et aux «éclipses de lune wŏlsik et dérèglements astraux sŏngbyŏn» (majoritaires). Les observations de phénomènes célestes considérés comme irréguliers y sont rapportées par catégorie et chronologiquement. Elles résultent de l'activité régulière et scrupuleuse ${ }^{44}$ des astrologues de la cour. L'abondance des notations s'explique par le choix des compilateurs de l'histoire officielle de rapporter de manière exhaustive tous les relevés de dérèglements astraux consignés dans les sources primaires comme critères d'évaluation de la politique des souverains de la dynastie précédente ${ }^{45}$. En complément, on trouve sporadiquement d'instructifs commentaires d'astrologues. Ces derniers étaient placés sous la surveillance des censeurs et faisaient l'objet de blâmes s'ils ne prédisaient pas les éclipses en temps et en heure.

\section{Le traité de Calendérologie ryŏk chi}

Selon l'introduction du traité de Calendérologie ${ }^{46}$, la cour du Koryŏ se serait contentée d'utiliser le calendrier Xuanming (823 ?) des Tang ${ }^{47}$, sans développer de théories particulières et sans tenir compte des multiples changements de calendriers (vingt-deux) que connut la dynastie des Song. La réforme la plus notable intervint sous le règne de Ch'ungsŏn par l'adoption du calendrier Shoushi des Yuan en $1281^{48}$, mais les astrologues auraient continué à se référer au précédent calendrier pour déterminer les dates des éclipses de soleil et de lune.

42. 天文志. L'ordre adopté dans le KRS n'est pas le même que celui du Songshi et du Yuanshi. Pyŏn 1982: 65.

43. Pyŏn 1982: 63.64 .

44. Pyŏn 1982: 65 (note 9).

45. $C f$. règles de compilation du KRS et du KRSCY (凡例: 災異之驗於事者 雖小必書 謹天譴也).

46. 曆志. KRS : 50,1a-b.

47. 宣明曆.

48. KRS : 29,30b. Par la suite, en 1298, l'astrologue Kang Po (姜保,?- ?) fut dépêché à la cour de Beijing pour se familiariser avec le nouveau calendrier. Il fut suivi en 1309 par l'envoi de Ch'oe Sŏng'il. En 1343, Kang Po rédigea le Susiryŏk ch'ŏpp ̌̆p ipsŏng (授 時曆捷法立成). 
Les notations du traité reprennent effectivement celles du Yuanshi ${ }^{49}$. En réalité, nous savons que d'autres calendriers impériaux furent introduits au Koryŏ, notamment le Jianxing des Song en $1022^{50}$ et le Datong des Ming en $1370^{51}$. En outre, le Dayan li (729) ${ }^{52}$ était bien connu des astrologues de Kaesŏng ${ }^{53}$, comme d'autres travaux divinatoires du moine Yixing $(673-727)^{54}$. En outre, il faut signaler qu'au cours de la dynastie furent élaborés des calendriers par les astrologues de la capitale coréenne afin de tenter de diminuer les écarts entre les théories et les observations, mais aussi de prévenir des dérèglements. En 1052, effectivement, Munjong ordonna aux membres du bureau de la Divination de rédiger cinq calendriers particuliers ${ }^{55}$ afin de prédire les infortunes à venir ${ }^{56}:$ un calendrier des Dix Essences sipchŏng, des Sept Astres ch'iljŏng, des Révolutions visibles hyŏnhaeng ${ }^{57}$, du Cycle caché tun'gap et du Grand Un T'aeil. De plus, on attribue en 1218 au président du bureau d'Astrologie Kim Tŏngmyŏng (?- ?) la rédaction d'un «nouveau calendrier» $\sin r y o{ }^{\circ}{ }^{58}$. Fort de ce constat, les historiens sud-coréens se sont depuis longtemps interrogés sur l'existence d'une culture calendérique propre au Koryŏ, développée dans le souci de réduire les erreurs des calendriers des Song en usage ${ }^{59}$. Les astrologues avaient en effet la possibilité d'effectuer et de consigner leurs propres observations des phénomènes célestes à partir d'un observatoire ch'ŏmsŏngdae situé à proximité du palais à l'intérieur

49. Pyŏn 1982: 67. Les spécialistes notent que les versions des deux calendriers chinois conservés au bureau de l'Astrologie comportent des différences avec les versions canoniques. CWS : 1451.6.15. Pyŏn 1982: 66-67.

50. 乾興曆. KRS : 4,38a. Rapporté de la cour de Kaifang par une légation du Koryŏ dirigée par Han Cho (韓祚, ?- ?), avec un traité de géomancie, le Shenghuifang yinyang erzheshu (聖惠方陰陽二宅書).

51. 大統曆. KRS : 42,6a.

52. 大衍曆. Cf. CWS : 1451.6.15.

53. KRS : 50,55a. Le Dayanli bingshu jieyao (大衍曆兵書接要) fait partie de la liste des quelques 125 ouvrages dont une copie fut demandée à la cour de Kaesŏng par l'empereur des Song en 1091 (KRS : 10,25b).

54. Cf. infra p. 20.

55. Pyŏn 1982: 69.

56. KRS: 7,22a (命太史...撰十精曆...七曜曆...見行曆... 遁甲曆... 太一曆 以禳來 歲災祥)

57. Quelques décennies plus tard, en 1100, le Conseil du palais réclama que l'auteur du calendrier fût relevé de sa charge en raison des erreurs du texte (KRS; 11,21b).

58. 新曆. KRSCY: 15,12b-13a.

59. Pak Sŏngnae cité par Pyŏn 1982: 69 (note 15). L'historien établit un lien entre la rédaction de calendriers autochtones et la mise en vigueur de noms d'ères propres à la dynastie à partir du XI ${ }^{\mathrm{e}}$ siècle. Kim Yongguk formule une thèse similaire (Kim, 1982 : 211). KRS : 9,33a-b. 
de l'enceinte royale ${ }^{60}$ dont la construction daterait du début de la dynastie ${ }^{61}$. Son usage fut considéré comme suffisamment important pour expliquer l'adoption en 1023 du terme sach'ŏndae en remplacement de t'aebokkam ${ }^{62}$.

Il est intéressant de noter dans le Koryŏsa la présence sous l'influence du bouddhisme d'éléments d'astrologie indienne tels que les «quatre astres invisibles» sa ŭm sŏng (dont Rahu et Ketu) ${ }^{63}$. Leur mention prend son sens dans le cadre de l'idéologie ritualiste du bouddhisme d'État qui accordait une grande place aux cérémonies originaires de la culture du bouddhisme ésotérique des Tang ${ }^{64}$. Ces astres sont ainsi mentionnés par exemple dans la «cérémonie d'extinction des calamités » sojae toryang ${ }^{65}$, un des rituels conjuratoires parmi les plus fréquemment mentionnés dans l'histoire dynastique. Connaissant l'idéologie anti-bouddhique notoire des compilateurs du Koryŏsa, il y a lieu de penser que les apports éventuels de la culture bouddhique à l'élaboration de théories et de pratiques originales autochtones furent négligés. Le même ouvrage mentionne pourtant en 1366 l'existence d'une carte du ciel sŏngsangdo dans un des plus prestigieux monastères de la capitale, le Pongsŏnsa, haut lieu du culte dynastique ${ }^{66}$.

\section{Le traité des Cinq agents ohaeng chi}

Le traité des Cinq agents est, lui aussi, largement inspiré de celui du Yuanshi. De plus, son introduction est fidèle à celle du même traité du Songshi. Une telle conformité marquerait la volonté des compilateurs néo-confucianistes d'appliquer la théorie de la «résonance entre le Ciel et l'Homme» ch'ŏnin

60. 瞻星臺. À l'Ouest de Manwŏldae (滿月臺; litt. «la Terrasse de la pleine lune») $c f$. Chunggyŏng chi 中京誌: k.7.

61. Kim 1982: 207. On a retrouvé les vestiges d'une structure en colonnes de pierre de 3m x 3m supposée construite sur le modèle de l'observatoire du Silla à Kyŏngju.

62. KRS : 76,44a. Kim 1982: 207.

63. KRS : 52,75a-b (四暗星). Les quatre astres (quatre émanations issues des grandes planètes) sont : émanation de Jupiter (紫氣), émanation de Mercure (月孛), nœud lunaire de Mars (羅㬋), nœud lunaire de Saturne (計都). Inclus dans le groupe des «neuf luminaires» (九曜); $c f$. KRS (salle des Neuf luminaires, 九曜堂): 9,34b; 13,29a; CWS : Sejong Chiriji: P'yŏngyanbu.

64. On attribue à Yixing plusieurs textes de rituels de conjuration des calamités astrales (T. $\mathrm{n}^{\mathrm{o}}$ 1304.1310).

65. 消災道場. 147 mentions dans les Annales de règnes du Koryŏ selon Sŏ Yun'gil (Sŏ 1993: 174). Selon Sørensen, le soutra de référence de ce rituel serait T. nº 964 (Sørensen: 2010).

66. 星象圖. KRS : 41,12b. 奉先寺. Monastère de vœu wŏnch'al 願刹 du fondateur de la dynastie. Han 1998: 289. 
kamŭng sŏl ${ }^{67}$. Dans le corps du traité, on trouve une compilation d'extraits des annales de règnes du Koryŏ se rapportant aux observations de phénomènes remarquables effectuées par les devins, classées par agent ${ }^{68}$. On comprend aisément que le principal enjeu de la compilation des dérèglements de la nature est idéologique: consigner les «blâmes du Ciel» pour mettre en évidence le degré de vertu de la politique des souverains ${ }^{69}$. L'étude de la fréquence des notations par règne montre la concomitance entre la fréquence des calamités (naturelles, humaines) et l'état de désordre de la politique à la cour ${ }^{70}$. Au cours de la dynastie, l'accroissement global et constant du nombre des observations appuie donc la thèse de la ruine morale du clan royal des Wang, contribuant à légitimer le changement de dynastie ${ }^{71}$.

\section{La littérature de référence de la divination d'État}

L'absence regrettable d'un catalogue des fonds des bibliothèques royales du Koryŏ ne permet pas une recension aisée des ouvrages utilisés par les astrologues et devins d'État. Pour en avoir un aperçu, il faut donc examiner les trois traités précédents, ainsi que certaines biographies reproduisant rapports ou dissertations à propos de matières divinatoires. De manière empirique, nous distinguons ici trois catégories de sources : les ouvrages importés de Chine, les écrits bouddhiques et la littérature autochtone.

\section{Les ouvrages importés de Chine}

La mention d'ouvrages de référence importés de Chine n'est pas fréquente. En plus des calendriers ${ }^{72}$, il convient d'ajouter les textes à caractère technique servant à évaluer les connaissances des candidats au concours de recrutement des fonctionnaires pour la section de divination et de géoscopie: le Xinji Dilijing, le Liushishu, le Dilijuejing, le Jingweiling, le Dijingjing, le Koushijue, le Taicangjing, le Hejue, le Xiaoshishu ${ }^{73}$, ainsi que les ouvrages dont de courts

67. 五行志. Pyŏn 1982: 70 (天人感應說).

68. Kim 2011: 15 .

69. Cf. supra note 45. Voir aussi KRS : 47,1a.

70. Les périodes caractérisées par la plus grande fréquence des phénomènes naturels irréguliers correspond à la période de prise de pouvoir des militaires (1170-1270) dont le régime de domination des Ch'oe d'Ubong (1219?-1268?) et les guerres contre les Mongols (1231-1270). Cf. Kim $2011: 49$.

71. Cf. Kim 2011: 49.

72. 元嘉曆. Kim 1992: 82. KRS: 108,11b.

73. KRS: 73,8a-b. Si l'on traduit littéralement les titres des ouvrages selon leur ordre de mention, cela donne: La Nouvelle compilation du Classique de géomancie; Le traité 
extraits sont reproduits dans les commentaires des devins et des astrologues présents dans les trois grands traités: le Hongfan wuxingzhuan yueling ${ }^{74}$, le Jingfang yizhuan ${ }^{75}$, le Tiandi ruixiang $z_{\text {hi }}{ }^{76}$, le Qian Hanshu $\mathrm{hu}^{77}$, le Wojing ${ }^{78}$, le Kaiyuanzhan $^{79}$, le Zhanshu ${ }^{80}$, le Bingzhi ${ }^{81}$, le Yinyang erzheshu ${ }^{82}$, le Yinyangshu ${ }^{83}$, le Zhenguan zhengyao ${ }^{84}$. Parmi la dizaine d'ouvrages recensés, on trouve une proportion de textes qui ne sont pas clairement identifiés (titres trop génériques), ou bien des ouvrages à caractère politique de portée générale.

\section{Les ouvrages bouddhiques}

Il est instructif de constater que les membres du bureau de la Divination du Koryŏ recommandaient explicitement la tenue de cérémonies bouddhiques pour conjurer les calamités naturelles et humaines ${ }^{85}$. De telles préconisations impliquaient une collaboration étroite avec les experts bouddhistes des affaires rituelles de l'État, à commencer par les membres du bureau des Affaires

de Maître Liu; Le Classique des méthodes de géomancie; Les ordonnances des trames et des chaînes; Le Classique du miroir de la Terre; Les recettes révélées de la tradition orale; Le Classique de l'inhumation des placentas; Recettes en rimes; Le traité de Maître Xiao.

74. 洪範五行傳月令. Litt. «Ordonnances mensuelles transmises selon les Cinq agents du Grand Plan». Yi 1984: 20.31.36.

75. 京房易傳. Litt. «Le Traité sur les mutations de Jing Fang». KRS : 10,23a；11,7b; $101,19 b$.

76. 天地瑞祥誌 (666) Litt. «Mémoire sur les signes fastes du Ciel-Terre». KRS : 53,54b. Cité 3 fois dans le KRS : 932, 1040, 1130. Selon certains spécialistes, l’ouvrage pourrait avoir été compilé par Sŏl Sujin 薛秀眞 (?- ?) du Silla (on attribue traditionnellement le texte au dashi Sa Shouzhen 大史 薩守眞). Il n'existe pas de trace de l'ouvrage en Chine, mais il est signalé au Japon en 876, puis en 928. Kwŏn Tŏgyŏng, en 1999, fait l'hypothèse de la transmission au Japon par la Corée (Kim 2001: 221).

77. 前漢書註. Litt. Annotation de l'Histoire des Han Antérieurs. KRS : 101,14a.

78. 握鏡. Litt. La Saisie du miroir. Le terme «saisie du miroir» est une expression métaphorique pour désigner la réception du mandat céleste par l'empereur. KRS : 54,35b.

79. 開元占. Abrégé du Tang Kaiyuan zhanjing (唐開元占經, 718). Litt. Classique de la divination de l'ère Kaiyuan des Tang. KRS: 54,24a.

80. 占書. Litt. Traité de divination. KRS : 53,47a.

81. 兵志. Litt. Traité sur l'armée. KRS : 55,25b.

82. 陰陽二宅書. Litt. Traité sur les deux demeures du yin et du yang. KRS : 4,38a.

83. 陰陽書. Litt. Traité sur le yin et le yang. KRS : 9,33b.

84. 貞觀政要. Litt. Essentiels de la politique de l'ère zhenguan. KRS : 55,3a.

85. En 1090, Ch'oe Sagyŏm (崔士謙; ?- ?), président du bureau d'Astrologie, envoyé en légation à la cour des Song, rapporta à Kaesŏng le protocole du Shuilu yiwen (水陸儀 文; litt. «Rituel pour la délivrance des êtres de la Terre et des Eaux»). 
bouddhiques sŭngnok $s a^{86}$. Dans le traité des Cinq agents, on trouve mention de soutras cités par les devins fonctionnaires pour conjurer les désordres: le Soutra des Rois bienveillants, le Soutra de l'Ornementation fleurie, le Soutra de l'Extinction des calamités, le Soutra du Diamant, la Dhâranî de l'Astre précieux ainsi que des soutras du bouddhisme ésotérique ${ }^{87}$. En fait, on peut supposer qu'en dehors de cultes périodiques funéraires dédiés aux membres de la famille royale, la plupart des rituels bouddhiques étaient exécutés avec la bénédiction des devins fonctionnaires: une pratique qui caractérise le ritualisme d'État de la cour ${ }^{88}$. La relation privilégiée des membres du bureau de la Divination au bouddhisme s'explique aussi parce que les édifices et sites bouddhiques (pagodes, cloches, statues) faisaient partie des catégories de bâtiments faisant l'objet de relevés par des fonctionnaires locaux, comme l'indique le traité des Cinq agents, impliquant une surveillance régulière des lieux concernés, effectuée par eux-mêmes ou des intermédiaires.

\section{Les ouvrages autochtones}

L'histoire officielle mentionne huit ouvrages autochtones qui relèvent de la littérature prophétique utilisée par les devins d'État. Celle-ci est caractérisée par des genres tels que les «notes secrètes » pirok ${ }^{89}$, pigi ${ }^{90}$, pisa, mais encore ch'amgi (litt. «écrits sur le destin du pays »). Parmi ces textes, la moitié environ semble avoir été héritée du Silla (Haedong kohyŏn ch'amgi ${ }^{91}$, Sinji pisa ${ }^{92}$ ); l'autre moitié rapporte les théories attribuées à Tosŏn, moine du Silla (828-897; alias Ongnyong, litt. «Dragon de jade»): Tosŏn milgi ${ }^{93}$, Tosŏn tapsan'ga ${ }^{94}$,

86. 僧錄司. Absent des traités administratifs. Cf. Hŏ 1993: 342-355.

87. La $4^{\mathrm{e}}$ lune de 1101, un rapport du bureau de la Divination prescrivit de recourir aux rituels de l'Abhiseka 灌頂, de Munduru 文豆婁, de l'Astre Précieux 寶星 pour conjurer le parasitage de la pinède du pic des Pins, Song'ak, montagne de protection chinsan 鎭山 de la capitale. En 1102, pour le même phénomène, ce fut la lecture du Soutra de l'Ornementation fleurie qui fut utilisée (KRS : 54,1b). En 1130, le bureau des devins recommanda la tenue d'une cérémonie bouddhique du Secours dans la détresse 度厄道場 pendant dix-sept jours pour éradiquer la présence d'un arc-en-ciel blanc (KRS : 54,42a).

88. 水陸儀文. KRS : 10,20b. Le culte est assez fréquent dans la liturgie du Xv siècle.

89. 秘錄.

90. 秘記. KRS : 54,39a.

91. 海東古賢讖記. Litt. «Récits prophétiques [sur le destin du pays] des anciens sages de l'Est de la Mer». KRS : 54,2b.

92. 神誌秘詞. Litt. «Compositions secrètes des traités divins». KRS : 122,2b. SGYS : 3, 興法, 寶藏奉老 普德移庵.

93. 道詵密記. KRS : 56,9b.58.15a.

94. 道詵踏山歌. Litt. «Chant d'excursion en montagne de Tosŏn». KRS : 122,1b. 
Ongnyonggi ${ }^{95}$. Elle témoigne ainsi de l'existence d'un courant de pensée actif au sein des devins fonctionnaires depuis le règne de Munjong, au milieu du $\mathrm{XI}^{\mathrm{e}}$ siècle ${ }^{96}$ et jusqu'à la fin de la dynastie. Dans ce courant, on trouve en particulier des genres propres à la littérature des maîtres de géomancie, de type tapsan ka «litt. «chants d'excursion dans les montagnes», ou encore myŏngdang $k i$ (litt. «écrits sur les palais lumineux mingdang ${ }^{97}{ }^{9}$. L'influence de ces textes reflète l'importance de la fortune politique des théories géomantiques au Koryŏ, à un niveau sans véritable équivalent en Asie orientale. En se référant à Tosŏn (associé au mythe de fondation de la dynastie dans le Koryŏsa dans des écrits des XII ${ }^{\mathrm{e}}$ et XIII ${ }^{\mathrm{e}}$ siècles), les théories s'appliquent à deux domaines principaux : les capitales et les édifices bouddhiques. Après la légitimation du choix du site de la nouvelle capitale (Kaesŏng) - en fait, le lieu d'origine du clan royal - se répand la thèse du déclin de l'énergie terrestre de la capitale royale et la nécessité d'y remédier; dans le même ordre d'idées, les édifices bouddhiques sont conçus comme devant pallier ou corriger (on parle de «géomancie de soutien de l'État» $p i b o^{98}$ ) les défauts géomantiques des sites du pays (à commencer par la capitale). Les théories sont finalement conçues par l'État comme un moyen de contrôler les constructions.

\section{La compilation des Notes secrètes du pays de l'Est de la Mer}

L'histoire de la divination en Chine est jalonnée d'étapes remarquables au cours desquelles furent réformés les traités en vigueur ${ }^{99}$. De la même manière, au début du règne de Yejong (r. 1105-1122) - un souverain féru de divination et de taoïsme - à partir de la $3^{\text {e }}$ lune de 1106 fut mis en place le «conseil temporaire pour l'épuration (des textes) » sanjŏng togam, composé d'une dizaine de conseillers confucianistes ainsi que de membres du bureau de Divination (non cités). Il compilèrent un volume à partir de différents traités du yin-yang et de géomancie chiri, qu'ils présentèrent au roi ${ }^{100}$ qui lui donna le titre de Haedong pirok (litt. «Notes secrètes du pays de l'Est de la Mer») ${ }^{101}$. Une édition de référence fut conservée à la Maison royale, d'autres exemplaires furent déposés

95. 玉龍記. Litt. «Récits du Dragon de jade». KRS : 72,11b.

96. KRSCY : 4,65a-66a; KRS : 56,5b (1056).

97. 三角山明堂記. Litt. «Écrits sur le palais lumineux du mont des Trois Cornes». KRS : 122,1a.

98. 裨補. Bruneton 2002: 31 .

99. À l'époque des Tang, Lü Cai (606-665) épura la littérature spécialisée et rédigea le Livre $d u$ Yin et $d u$ Yang 陰陽書. Cf. Xin Tangshu 新唐書: 107 (呂才傳).

100. 刪定都監. KRS : 12,21a (命儒臣與太史官...刚定陰陽地理諸家書 編一冊以進).

101. 海東秘錄. Deux mentions dans le KRS (KRS : 96,11a). 
à la cour du palais, au bureau de l'Astrologie et au bureau de la Divination ${ }^{102}$. Par la suite, il n'est pratiquement plus fait mention de l'ouvrage dans l'histoire dynastique.

Au début du Chosŏn, en 1394, une compilation/épuration des ouvrages de divination, le (Chiri) Pirok ch’waryo (litt. «Contenus essentiels sélectionnés des notes secrètes »), fut réalisée. Puis, la même année, un conseil procéda à un nouveau tri des ouvrages divinatoires de la précédente dynastie conservés dans les fonds du bureau de la Divination ${ }^{103}$. Si l'on ajoute à ces mesures la destruction de la quasi-totalité de la littérature prophétique du Koryŏ ordonnée par T'aejong ${ }^{104}$, on peut estimer qu'au cours du Xv ${ }^{\mathrm{e}}$ siècle - à l'exception de bribes citées dans les sources officielles - s'éteignit durablement la mémoire de la culture divinatoire des Wang.

Les quelques clés nécessaires à la compréhension des rouages de la divination officielle du Koryŏ, que nous venons d'examiner, nous permettent à présent de situer et d'interpréter les activités des devins et astrologues impliqués dans son fonctionnement.

\section{Les acteurs de la divination du Koryŏ}

Dans le Koryŏsa, la terminologie utilisée pour désigner devins et astrologues est souvent imprécise ${ }^{105}$. Elle constitue donc un obstacle fondamental à la compréhension des relations entre, d'une part, les pratiques privées et étatiques de la divination, et, d'autre part, leur caractère laïque ou religieux. Autrement dit, il est souvent difficile d'identifier les acteurs de la divination ainsi que leur statut administratif et social. En outre, quand il s'agit de mentionner les devins fonctionnaires en tant que groupe (communiquant leurs rapports à la hiérarchie), l'anonymat domine. Entre l'émiettement que produisent les citations

102. KRS : 12,21a; KRSCY : 7,12b. Il semble que la pratique du dépôt d'ouvrages de divination importants dans plusieurs administrations était ordinaire (voir par exemple KRS : $127,28 b)$.

103. 秘錄撮要.CWS : 1393.12.11; 1394.2.14.16; 1394.7.11-12. Un rescrit du conseil général des Délibérations précisa que des «notes secrètes » pirok (秘錄) avaient été transmises de l'ancienne dynastie et que les théories y étaient confuses et non concordantes. Le lendemain, un «conseil temporaire d'épuration du yin-yang»(陰陽刪定都監) fut mis en place, composé de neuf conseillers et de membres du bureau de la Divination. Il est précisé que les ouvrages examinés étaient des traités de géomancie relatifs aux prophéties.

104. CWS : 1417.12 .15 .

105. En dehors des noms de charges, les termes indifférenciés employés sont par exemple ilcha (日者), ilgwan (日官), sulcha (術者), sulsa (術士), surin (術人), pokcha (卜者), ŭmyang ka (陰陽家). 
sporadiques d'individus et l'opacité des mentions collectives rapportées «en bloc», la difficulté est extrême à saisir la nature des associations et collaborations au sein de la divination d'État. Un traitement aussi négligé s'explique par un ensemble de causes relevant de l'état des sources et de l'idéologie des historiographes. En dehors de l'histoire officielle, les apports de l'épigraphie (épigraphie bouddhique, épitaphes enterrées) et des sources à caractère privé, comme les recueils d'œuvres de lettrés (il en existe environ une quarantaine pour la période qui nous intéresse), sont peu abondants, et, sauf exception, ne sont guère plus précis. J'ai choisi de présenter ici une typologie empirique des devins du Koryŏ en distinguant les acteurs de la divination appliquée à des enjeux politiques et les praticiens de la divination privée : ils représentent deux groupes sociaux distincts (mais non mutuellement exclusifs), traités de manière contrastée dans le Koryŏsa.

\section{Les acteurs politiques}

Les difficultés inhérentes à la source que nous analysons, conjuguées à la diversité des enjeux politiques impliquant le recours à la divination au cours de la période étudiée, rendent complexe la présentation des acteurs de la divination d'État. Aussi est-il nécessaire de procéder à différents niveaux d'analyse, dont les plans s'articulent en fonction de leur rapport à l'idéologie politique du Koryŏsa, en commençant par les aspects les plus explicites. Dans un premier temps, nous examinerons le lieu par excellence de mise en valeur des devins dans le genre de l'histoire dynastique : les biographies des spécialistes panggi (section des biographies). Ensuite, nous porterons notre attention sur les liens étroits entre enjeux politiques, théories divinatoires et statut administratif des devins à travers l'analyse de figures emblématiques. Enfin, nous nous intéresserons à l'aspect sans doute le plus original de la divination officielle du Koryŏ : l'école de Tosŏn, et à la problématique connexe de la transmission des savoirs de la culture divinatoire de la Corée médiévale.

\section{Les personnalités mises en valeur dans les biographies des spécialistes}

Dans le Koryŏsa, il existe peu de personnalités mises en valeur pour leur activité en matière de divination. Dans la catégorie des «spécialistes » panggi ${ }^{106}$, on trouve seulement cinq exemples dont deux devins ${ }^{107}$. La première biographie,

106. Les biographies des «spécialistes» se trouvent au kwŏn 122 du KRS, juste avant la première catégorie négative des Biographies: les «Eunuques» hwanja 宦者.

107. Les autres exemples sont un peintre (Yi Nyŏng 李寧, ?- ?; XII ${ }^{\mathrm{e}}$ s.) et deux médecins (Yi Sangno 李商老,?-?, XII ${ }^{\mathrm{e}}$ s.; Sŏl Kyŏngsŏng 薛景成, 1237-1313). 
celle de Kim Wije (?- ?) ${ }^{108}$ est constituée d'extraits du rapport que ce dernier communiqua au roi Sukchong (r. 1095-1105) en 1096 pour demander le transfert de la capitale principale dans la capitale du Sud (Yangju) ${ }^{109}$. Il ne s'agit donc pas en réalité d'un texte biographique. Le personnage, en 1096, n'était pas membre du bureau de la Divination mais assimilé assistant au conseil des Insignes, fonctionnaire de sixième échelon inférieur. Cependant, il est précisé qu'il bénéficiait du soutien du devin fonctionnaire ilcha Mun Sang (?-?), en deuxième position au sommet de la hiérarchie des devins d'État ${ }^{110}$. Kim Wije représente ainsi un cas peu courant de fonctionnaire non affilié aux bureaux spécialisés de la divination dont l'initiative - en l'occurrence la communication d'un rapport au souverain portant sur des matières divinatoires - trouva crédit à la cour, grâce à la complicité des dirigeants du bureau de la Divination. En effet, quelques années après l'émission du document, une procédure se mit en place, aboutissant à l'instauration du «Conseil temporaire de la fondation de la capitale du Sud», qui fut chargé de trouver un site pour la nouvelle capitale secondaire ${ }^{111}$. La biographie de Kim Wije est en définitive un texte d'une importance cruciale pour la compréhension de la divination d'État du Koryŏ puisqu'il représente un condensé unique des théories géomantiques en vigueur à la cour, comportant la plus grande densité de références à la littérature prophétique de Tosŏn. Par ailleurs, il était sans doute important pour les compilateurs du $\mathrm{XV}^{\mathrm{e}}$ siècle de mettre ainsi indirectement en valeur le site de la capitale du Sud du Koryŏ en raison de sa proximité avec celui de la future (et unique) capitale du Chosŏn ${ }^{112}$.

La quatrième biographie est celle d'O Yunbu (?-1304), le second devin de la catégorie. Sous le règne de Ch'ungnyŏl (r. 1274-1298.1299-1308), celui-ci occupa la charge de président du bureau de l'Observation du climat kwanhusŏ. On dit qu'il excellait dans la prédiction du caractère faste des phénomènes célestes et terrestres chŏmhu et dans les pronostics par le tirage des tiges

108. 金謂磾. On trouvera une traduction en français de la Biographie de Kim Wije en appendice de Bruneton 1997 ( $c f$. bibliographie).

109. 南京 Namgyŏng à Yangju 楊州. KRS : 56,9a-10a.

110. 日者文象. La même année, il est cité comme directeur provisoire du bureau de la Divination et premier conseiller du bureau d'Astrologie (攝司天監知太史局事; HKC $\mathrm{n}^{\circ} 231$, p. 546). Mun Sang est mentionné en 1101 comme directeur de la Petite intendance (charge de 4 échelon supérieur) à la retraite et participant au conseil temporaire de la fondation de la capitale du Sud (KRS: 11,31a). Si l'on considère que sa charge de retraite constitue le point culminant de sa carrière, on en déduit qu'il devait occuper un poste de devin d'échelon inférieur: directeur ryŏng 令 ou kam 監.

111. 南京開創都監. KRSCY: 6, 40b-41a.KRS: 11,31a-b.

112. Bruneton $1997: 253$. 
d'achillée $p o k s \breve{o}^{113}$. Sa réputation fut renforcée par le fait qu'il fut convoqué par Kubilai (r. 1260-1271, alias empereur Shizu des Yuan) pour être mis à l'épreuve de son art. Il réalisa une carte du Ciel pour le souverain, que les astrologues prirent tous pour modèle par la suite. Le roi le comparait à Cui Hao (?-450) qui s'illustra dans l'histoire des Wei du Nord. En outre, il recommanda la tenue de cérémonies bouddhiques pour répondre aux «blâmes du Ciel».

L'ensemble des éléments plus ou moins décousus décrivant la carrière de ce devin modèle ne sont pas explicitement liés à des enjeux politiques majeurs. Certes, on ne peut nier qu'il incarne un certain esprit de résistance à la domination mongole en prenant, à plusieurs reprises, position contre l'intervention de la reine Wŏnsŏng (1259-1297), originaire de la cour des Yuan et fille de Kubilai, sur des sujets concernant les rites dans le Temple des ancêtres de la dynastie, les cérémonies conjuratoires et la construction de palais, n'hésitant pas pour cela à y sacrifier sa position et s'attirer les foudres de l'intéressée. La biographie d'O Yunbu s'inscrit en définitive dans la tradition historiographique du genre: montrant un modèle de devin incorruptible et loyal ${ }^{114}$, mais son rôle politique le distingue de la plupart des autres figures de devins du Koryŏsa, telles que celles que nous présentons maintenant.

\section{Enjeux politiques, théories et statuts des devins}

Dans l'histoire officielle, évaluer le rôle des devins et leur position à la cour nécessite d'analyser l'articulation entre les enjeux politiques liés aux domaines d'application de leurs compétences, les théories qu'ils préconisent, ainsi que leur statut administratif. Je propose ici de décrire plusieurs exemples remarquables de devins autour de trois enjeux cruciaux pour le clan régnant: la légitimation du pouvoir, la protection du pays contre les calamités, la prolongation de la durée de la dynastie.

\section{Légitimer le pouvoir en place}

L'utilisation d'arguments divinatoires pour légitimer le pouvoir en place est un phénomène qui se manifesta à au moins deux reprises dans l'histoire du Koryŏ : 1) au moment de l'instauration de la dynastie des Wang, au début du $\mathrm{X}^{\mathrm{e}}$ siècle, et 2) pendant la période de la domination du clan des Ch'oe d'Ubong (1196-1258), à partir de Ch'oe Ch'unghŏn (1149-1219) et jusqu'à la quatrième génération de ses descendants. La problématique particulière de la légitimation

113. 伍允孚. KRS: $122,5 b-8 b$ (精於占候... 又善卜筮).

114. La description d'O Yunbu est conforme aux stéréotypes des devins de la tradition historiographie chinoise (laideur, obstination, sincérité, fidélité au souverain). 
du pouvoir par la divination suppose des relations étroites entre devins et souverains: les premiers étaient récompensés soit par l'octroi d'une charge de devin soit par le statut spécial de conseiller privé (favori) du roi.

$\mathrm{Du}$ début du $\mathrm{x}^{\mathrm{e}}$ siècle, nous ne connaissons guère comme devins que la figure de Ch'oe Ch'ongjin (907-987), plus connu sous le nom de Chimong (litt. «connaisseur des rêves»), que lui attribua le fondateur de la dynastie. S'il ne figure pas parmi les «spécialistes», c'est vraisemblablement parce que sa biographie officielle a été classée dans la catégorie des biographies des «sujets méritants de fondation du pays». On le dit expert en matière de connaissance des signes du Ciel et de pronostics par tirage des tiges d'achillée ${ }^{115}$. Faisant partie de l'escorte rapprochée de Wang Kŏn, il lui prédit en 936 qu'il gouvernerait les Trois Han ${ }^{116}$. Il compte parmi les tout premiers devins fonctionnaires de la nouvelle dynastie à être cités dans le Koryŏsa. Membre du bureau de l'Astrologie sach'ŏn kwan en 945, il prédit le complot de Wang Kyu (?-945) en observant une étoile filante pénétrer dans la zone de la Ténuité pourpre (ziwei), puis en tirant les sorts. Chŏngjong (r. 946-949) le nomma conseiller d'État. Sous Kyŏngjong (r. 975-981), il prédit encore le complot de Wang Sŭng (?-?) en annonçant au roi qu'un «astre étranger était entré irrégulièrement dans la constellation impériale». En définitive, il joua un rôle important de légitimisation et de consolidation de la dynastie pendant les premiers règnes du Koryŏ.

Au cours de la «période de prise de pouvoir par les militaires» (11701270), à la suite de son père Ch'oe Ch'unghŏn, Ch'oe I (?-1249) se distingua par l'emploi de devins tels que l'ancien moine Chu Yŏnji ${ }^{117}$, renommé Ch'oe Sanbo (?-1127), et son disciple To'il (?-1227) ${ }^{118}$, un «moine spécialiste» sulsŭng. En effet, après sa venue dans la capitale, Ch'oe Sanbo utilisa ses compétences en divination chŏmsul pour s'attirer les faveurs des puissants, dont celle de l'homme fort du régime. Ch'oe I prit l'habitude de le consulter sur tous les sujets, si bien que l'influence du devin augmenta à la cour. En 1227, le devin lui annonça secrètement que sa physionomie lui prédisait pour destin de prendre la place du roi. L'affaire fut ébruitée de sorte que Ch'oe Sanbo et To'il furent exilés puis noyés. L'histoire officielle ne faisant pas état du statut administratif des deux personnages, il n'est pas possible d'affirmer qu'ils étaient rattachés au bureau de la Divination.

115. 崔知夢 (崔聰進). KRS : 92,12b-14a (精於天文卜筮).

116. 三韓. Expression désignant l'ensemble de la péninsule coréenne (territoire), sa population, sa culture, sans référence politique (voir par exemple: KRS : 81,1a).

117. 周演之 (崔山甫). KRS : $101,25 \mathrm{a} ; 102,12 \mathrm{~b} ; 129,32 \mathrm{~b}$.

118. 道一. KRS: $129,33 \mathrm{a}$. 


\section{Protéger le pays des calamités}

L'idéologie ritualiste de la dynastie des Wang, qui s'appuyait en partie sur une conception magique du bouddhisme, produisit un imposant dispositif de cultes bouddhiques de conjuration, dont une proportion importante relevait de pratiques du milgyo, le bouddhisme ésotérique. Elle supposait de ce fait une collaboration étroite entre les spécialistes des cultes et devins fonctionnaires, qui se retrouvaient chargés de missions similaires du point de vue de l'État. Au cours du Koryŏ, il n'est donc pas étonnant de trouver certains moines dont le statut combinait, semble-t-il, les deux types de compétences. À partir du XII ${ }^{\mathrm{e}}$ siècle, le Koryŏsa rapporte l'existence de religieux ou de devins incitant les souverains (Ǔijong : r. 1146-1170, Myŏngjong : r. 1170-1197) à pousser la logique ritualiste à un niveau accru: soit en augmentant la fréquence des cérémonies périodiques existantes, soit en diversifiant le type de rituels, soit en recommandant leur exécution par anticipation, à titre préventif. Le moine Ch'isun et le devin Yŏng Ŭi en sont deux exemples emblématiques.

Yŏng Ŭi (?-1170), qualifié par les historiographes de devin pok cha, fit son apparition à partir de 1152, en tant que membre d'un groupe de fonctionnaires dont l'expulsion était réclamée par plusieurs censeurs ${ }^{119}$. En 1157, il est mentionné comme intendant et devin, mais son appartenance au bureau de la Divination n'est pas précisée. Toujours est-il qu'il prôna la théorie des cérémonies de conjuration yanghoe et que le souverain y accorda crédit ${ }^{120}$. Pour le devin, la durée de vie de la dynastie ainsi que la longévité du roi dépendaient de l'assiduité à pratiquer les prières de conjuration, et de la fréquence des tournées d'inspection royale. Il effrayait Ǔijong avec l'annonce de calamités à venir pour justifier la mise en place de rites bouddhiques. Sur ses recommandations, le roi fit réaliser de nombreuses images d'Indra et d'Avalokiteshvara, qui furent diffusées dans les monastères de la capitale et des provinces. En tant que progéniture de rebelle, sa carrière était limitée, mais le roi fit corriger sa généalogie. Yŏng Ŭi fut assassiné avec d'autres favoris du roi au moment de la révolte de 1170.

À partir de la prise de pouvoir des militaires, l'histoire dynastique rapporte l'existence d'une nouvelle catégorie de religieux: les «moines spécialistes» sulsŭng ${ }^{121}$, particulièrement influents auprès du clan des Ch'oe. Le plus important d'entre eux fut Ch'isun (?-?): sous le règne de Myŏngjong, il persuada d'abord la cour de procéder à des cérémonies conjuratoires dont les dépenses seraient prélevées sur les appointements des fonctionnaires ${ }^{122}$. À cette

119. 卜者 榮儀. Sa biographie est en KRS : 123,2a-3b. KRSCY : 11,14a.

120. 禳禬. KRS: 123,2a-3b.

121. 術僧. 7 mentions dans le KRS.

122. 致純. KRS : 77,26a-b. 
occasion, en 1178, fut instauré un «conseil temporaire des prières extraordinaires pour les bienfaits ${ }^{123}$. Ensuite, l'année suivante, il obtint d'organiser à titre extraordinaire ${ }^{124}$ la cérémonie des Cent sièges des Rois bienveillants pour conjurer les effets néfastes des révoltes armées des années précédentes ${ }^{125}$. L'influence du moine suggère qu'il occupait une place importante et qu'il recevait l'appui des devins fonctionnaires. Je fais donc l'hypothèse qu'il était rattaché au bureau des Affaires bouddhiques, avec une éventuelle charge cumulée au bureau d'Astrologie, justifiant ses compétences à la fois dans le domaine des rites bouddhiques et dans celui de la divination.

Prolonger la durée de la dynastie par le déplacement du roi

À partir du milieu du $\mathrm{XI}^{\mathrm{e}}$ siècle, l'histoire officielle mentionne l'apparition de théories inédites au sein de la divination d'État. Celles-ci s'appuient sur la littérature prophétique de Tosŏn. La thèse centrale de l'ensemble de cette littérature consiste en la croyance dans le déclin à plus ou moins brève échéance de l'énergie terrestre chigi (ou énergie royale wanggi) ${ }^{126}$ de la capitale principale du Koryŏ, et dans la nécessité, pour y remédier, de faire se déplacer le souverain (physiquement ou symboliquement), temporairement ou définitivement, dans un autre lieu. Par l'expression yŏn'gi ${ }^{127}$, le Koryŏsa décrit des remèdes à un tel déclin, conçus par des générations de devins plus ou moins officiels. On comprend dans ces conditions que l'histoire de la divination au Koryŏ est caractérisée par le poids de la géomancie - l'art du choix des sites des demeures (palais) - qui sous-tend une telle idéologie, ainsi que par l'originalité des applications politiques des théories, qui donnèrent lieu à une géomancie des capitales. À partir du règne de Munjong et jusqu'à celui d'Injong (1056-1135), la géomancie des capitales conduisit à fonder deux capitales secondaires : celle du Sud et celle de l'Est, en plus de la capitale de l'Ouest préexistante. La période est marquée par la fondation de la capitale du Sud (deux tentatives en 1056 et en 1101), puis par la révolte de la capitale de l'Ouest de $1135^{128}$. Toutes les

123. 別例祈恩都監. KRS: 77,26a-b; KRSCY: 12,36b.

124. La cérémonie des Cent sièges était censée être exécutée une fois tous les trois ans selon les codes.

125. KRS : $20,3 a$.

126. 地氣, 王氣.

127. 延基(業). Littéralement: «prolongement de la base (de l'œuvre dynastique)». Le terme est relativement fréquent dans les sources (13 mentions dans le KRS). Il est parfois également question de «site de restauration (de la dynastie)» chunghŭng chi chi 重興 之地. KRSCY: 11,27b.

128. Les historiens contemporains le désignent généralement par l'expression «Révolte de Myoch'ŏng» (Myoch'ŏng-ŭi nan 妙淸의 亂), une formulation mal venue en ce qu'elle 
fondations débutent par la sélection du site d'un palais secondaire, susceptible d'accueillir le souverain. La révolte de 1135 éclata à l'issue de plusieurs années d'efforts d'une faction régionaliste à la cour ${ }^{129}$ pour persuader le roi de transférer la capitale principale à P'yŏngyang. Selon le Koryŏsa, deux devins de la cité de l'Ouest y jouèrent un rôle majeur.

Le duo formé par le moine Myoch'ŏng (?-1135) et son disciple Paek Suhan (?-1135) incarne symboliquement l'étroitesse des liens entre bouddhisme et divination ${ }^{130}$, mais aussi et surtout, la fidélité aux théories de Tosŏn, relayée par l'école de divination de P'yŏngyang ( $c f$. supra). À ce titre, ils trouvèrent du crédit auprès d'une partie des tenants de la divination à la cour de Kaegyŏng, sans toutefois y faire l'unanimité. Pour s'en persuader, il suffit d'observer le nombre de calamités relevées dans le traité des Cinq agents correspondant à la période d'influence de Myoch'ŏng et de ses partisans (1128-1135), ainsi que les commentaires des astrologues ${ }^{131}$. De la vie de Myoch'ŏng et de son comparse, nous ne savons à peu près rien ; cependant, à l'apogée de son influence à la cour, en 1134, Myoch'ŏng reçut un habit violet et fut nommé premier conseiller au bureau de la Clepsydre (de la capitale de l'Ouest), montrant par là que, outre la reconnaissance de son statut de devin fonctionnaire, il existait dans certains cas la possibilité pour un religieux d'être nommé à une charge du bureau de la Divination. Quant à Paek Suhan, il semble qu'il fût intégré au bureau de la Divination de la capitale principale après avoir été devin de P'yŏngyang.

À propos de l'école bouddhique d'affiliation du moine, les thèses des historiens se bornent à des conjectures. Myoch'ŏng se fit d'abord connaître comme promoteur de rituels ésotériques (cérémonies d'Âtavaka et d'Aparâjita). En 1132, il officia lors de la cérémonie d'ouverture des travaux pour la reconstruction du palais royal de la capitale principale en exécutant la technique

fait porter de manière excessive le poids de la responsabilité à un individu.

129. La faction était formée en partie de hauts fonctionnaires originaires de P'yŏngyang, et représentée par Chŏng Chisang 鄭知常 (?-1135), Kim An 金安 (?-1135), Mun Kong'in 文公仁 (conseiller d'État;?-1137), Im Kyŏngch’ŏng 林景淸 (président de la Cour centrale du gouvernement; ?- ?), Hong Isŏ 洪粦敍 (conseiller d'État;?-?), Yi Chungbu 李中孚 (?-?).

130. 白壽翰. KRS : 127, 26b-36a. Les données proprement biographiques relatives aux deux personnages sont maigres. L'association entre bouddhisme et divination est recherchée par les compilateurs de l'histoire officielle pour démontrer le caractère funeste de leur combinaison pour le destin de la dynastie. On trouve une traduction annotée de la biographie de Myoch'ŏng dans Bruneton 2002: 343-357.

131. Parmi les phénomènes néfastes qui se produisirent, on note les foudroiements de la pagode de neuf étages du monastère de Chunghŭng de la capitale de l'Ouest auquel le moine était rattaché $(1129.9 ; 1130.9)$, ainsi qu'un déchaînement d'intempéries lors d'un voyage du roi qui se rendait à P'yŏngyang. KRS : 54,2b.31a.42a; 55,4b.10a-b. 
dite de la «Marche de la tente de jade du Grand Un» ${ }^{132}$ : un procédé censé conférer l'invincibilité, s'apparentant aux méthodes du Cycle caché tun'gapsul ${ }^{133}$. Par de telles pratiques, Myoch’ŏng présente plusieurs points communs avec une catégorie de praticiens mentionnée seulement au début du Xv siècle (1401, 1417-1419) sous la désignation de mirwŏn ( «officiants ésotériques ») rattachés à l'école du bouddhisme ésotérique du Ch'ongji ${ }^{134}$, dont on peut supposer qu'ils existèrent au Koryŏ.

Les deux devins de la capitale de l'Ouest furent exécutés après l'éclatement de la révolte, l'un dans la capitale secondaire, l'autre, à Kaegyŏng. De plus, il semble que l'école de divination de la cité de l'Ouest fut dissoute par la suite. Ainsi, P'yŏngyang fut durablement disqualifiée comme site de remplacement de la capitale royale. Toutefois, dès le règne suivant (Ŭijong), les devins préconisèrent d'autres déplacements royaux, mais au moyen de formulations nouvelles. Yŏng Ŭi, cité précédemment, recommanda en 1157 la construction d'un palais secondaire dans la province du Nord-Est (Kwandong) ${ }^{135}$. Dans le même ordre d'idées, en 1158 , le préposé à la température du bureau de la Divination, Yu Wŏndo (?-?), fit la promotion d'un site dans les monts T'o à Paekchu afin de mater les «Barbares du Nord» pungno dans l'espace de sept années ${ }^{136}$. Le conseiller d'État Ch'oe Yunŭi (1102-1162) fut alors dépêché sur place pour examiner la configuration géomantique p'ungsu du lieu. Finalement le palais Taehwa fut bâti en un temps record, mais il suscita la critique de certains experts sulcha, férus des thèses de Tosŏn. L'inauguration des bâtiments fut ponctuée de signes néfastes faisant douter le roi de l'efficacité de la méthode.

À la théorie des écrits de Tosŏn préconisant la mise en place d'un système de tournées saisonnières dans trois capitales, fut alors substitué un avatar: la théorie dite des Trois So (So de gauche, de droite et du Nord), ou lieux de régénération (de l'énergie terrestre), apparue à partir de $1174^{137}$, et réactualisée jusqu'en 1379. Elle marque les règnes de Kojong (r. 1213-1259), Ch'ungnyŏl, Kongmin et U. De plus, sous le long règne de Kojong (1218, 1228, 1234, 1235, 1259), fut reprise l'idée inaugurée par Myoch'ŏng qu'il était possible de pallier la présence royale par la déposition ritualisée de la tenue royale dans les palais édifiés sur les sites favorables. Il n'est pas possible ici d'énumérer tous les astrologues et devins mentionnés dans l'histoire officielle pendant cette période.

132. 太一玉帳步法. KRS : 127,30a.

133. 遁甲術.

134. 密員 (摠持宗密員). Bruneton 2002: 376 .

135. KRSCY: $11,21 \mathrm{a}$.

136. KRS : 18,11b-12a (太史監候劉元度奏 白州兔山半月岡 實我重興之地 若營宮關 七年之內可吞北虜).

137. 三蘇. KRSCY: 12,12a. 
Je retiens les cas les plus remarquables et les plus ambigus du règne de Kojong retenus par les historiographes.

Yi Chisik (?- ?), expert surin, ainsi que Kim Tŏngmyŏng (?-?) firent une première apparition en 1217, par l'entremise de Ch'oe Ch'unghŏn, l'homme fort du régime, attentif aux conseils des devins dans un contexte politique instable ${ }^{138}$. Le premier, de statut inconnu, semble avoir exercé une influence considérable en matière de divination. Le second, la même année, se préoccupa de conjuration militaire par la géomancie ${ }^{139}$, mais sans avoir de position établie au sein de l'appareil d'État, si l'on considère le manque de coopération du bureau d'Astrologie pour appliquer ses recommandations. Il provoqua ainsi l'exil du président du bureau d'Astrologie, Ch'oe Kyeryang (?- ?), auquel il succéda rapidement. Un devin anonyme sulsa (Yi Chisik?) préconisa alors le séjour du roi dans un palais secondaire pour renflouer l'énergie royale de la capitale principale. À la fin de 1217, Yi Chisik prôna différents types de mesures: la destruction d'un monastère pour conjurer les armées ennemies, le déplacement de la tablette funéraire de Sŏngjong dans un autre «monastère de vœux ${ }^{140}$ », la construction d'un nouveau palais au pic Paek, et le déplacement du roi dans le monastère de Hyŏnsŏng, centre de la lignée spirituelle de Myŏngnang (école du Sceau sacré) ${ }^{141}$. Au vu de l'ensemble de ces faits, on peut présumer, à l'instar d'un Myoch'ŏng, que le devin était proche des milieux pratiquant les rituels ésotériques bouddhiques. Rétrospectivement, ce fut vraisemblablement sous son instigation qu'au cours de l'année écoulée, l'on exécuta les rituels de Muduru ${ }^{142}$, d'Aparâjitâ ${ }^{143}$, et de Marichi ${ }^{144}$.

En 1218, Kim Tŏngmyŏng présenta au roi son «nouveau » calendrier. Les historiographes précisent alors que le personnage était à l'origine un religieux, qui proférait des propos inconsidérés sur le yin et le yang, mais qu'ayant trouvé faveur auprès de Ch'oe Ch'unghŏn, qui l'avait nommé à ce poste, il ne se trouva

138. Le régime des Ch'oe d'Ubong (1196-1258) faisait l'objet de menaces à l'intérieur et à l'extérieur du pays.

139. Yi Chisik expliqua à Ch'oe Ch'unghŏn que les troubles armés s'expliquaient par la position de la tombe $\mathrm{Hu}$ (厚陵), mais le «conseil temporaire pour le déplacement de la tombe $\mathrm{Hu} »$ fut dissous peu après (KRS : 22,9b-10a).

140. Les versions du KRS et du KRSCY suggèrent deux interprétations possibles concernant l'attribution à Yi Chisik du déplacement des tablettes. Quant aux déplacements de Kojong dans les monastères, il s'inscrivaient dans la continuité avec les pratiques de l'année précédente.

141. SGYS : 5 (神呪, 明朗神印).

142. KRS : 22,11a.14b.

143. KRS : $22,10 b .13 b$.

144. KRSCY : $15,7 \mathrm{~b}$; KRS : $22,11 \mathrm{~b}$. 
ni astrologues ni censeurs pour le contester (le calendrier en question aurait été truffé d'erreurs) ${ }^{145}$. Parallèlement à la nomination de Kim Tŏngmyŏng, tout porte à croire que l'influence de Yi Chisik se poursuivit, présumant d'une collusion entre les deux personnages. La $9^{\mathrm{e}}$ lune, il aurait prescrit au roi de donner sa tenue militaire pour la déposer dans un sanctuaire en vue de conjurer les armées Khitan ${ }^{146}$. En 1228, il est encore indirectement question de lui, puisqu'un membre du Conseil secret fut envoyé dans le palais secondaire du pic Paek $^{147}$, porteur de l'habit et de la ceinture royaux, dans la ligne des directives de l'ancien devin dont il n'est plus fait mention. En 1234, on assiste à la reprise, au moins partielle, de théories semblables, suscitée par l'influence d'un moine inconnu. Ce dernier réactualisa la théorie des Trois So en affirmant que le site de Yangju (la capitale du Sud) correspondait à Asadal, le So de gauche, qu'il fallait y construire un palais pour que le roi s'y rende et prolonge ainsi de huit siècles la fortune du pays. De fait, au cours de la $7^{\mathrm{e}}$ lune, l'eunuque Yi Paekchŏn se rendit dans le palais de la capitale du Sud pour y déposer l'habit royal ${ }^{148}$.

La fin du règne de Kojong est marquée par l'activité d'un nouveau devin, Paek Sŭnghyŏn (?- ?), mentionné dans la catégorie négative des «favoris » ${ }^{149}$ et dont on dit qu'il vivait de la pratique de la géomancie ${ }^{150}$. En 1259, ce fut en tant que colonel remplaçant ${ }^{151}$ qu'il fut sollicité par le roi pour définir des sites susceptibles de prolonger la durée de vie de la dynastie. Il préconisa que le souverain se déplaçât dans le monastère Hyŏlgu de Kanghwa pour y débattre du Soutra du Lotus, puis de construire un palais à Samnangsŏng (litt. « fort des Trois Gentilhommes ») afin d'en vérifier les effets positifs. Les deux conseils du gouvernement s'en tinrent à l'avis du président du bureau d'Astrologie, An Pangnyŏl (?-1271), quant au bien fondé de l'entreprise. Après une discussion interminable entre Paek Sŭnghyŏn et le devin fonctionnaire, ce dernier finit par recommander de tester provisoirement la méthode, bien qu'il ne la considérait pas comme crédible. Les biographes ajoutent que le géomancien se nourrissait des lectures de textes taoïques, bouddhiques, divinatoires et prophétiques pour justifier ses théories. Il mettait en avant la croyance, répandue au Koryŏ, selon laquelle les sites de la péninsule coréenne correspondaient aux lieux de séjour permanents des divinités bouddhiques (Bouddhas et êtres d'Éveil),

145. KRSCY : 15,13a (所進新曆 率皆任意變更古法). Le passage est absent du KRS.

146. KRS : 22,15b-16a; KRSCY: $15,15 a$.

147. 白岳. TYS : $12,2 \mathrm{~b}$ (長湍都護府). KRSCY: 15,44a.

148. KRS : $23,28 \mathrm{a}-\mathrm{b}$; KRSCY : $16,21 \mathrm{~b}$.

149. KRS: $123,5 b$.

150. 白勝賢. KRS : $123,5 \mathrm{~b}$ (業風水).

151. 補郎將. Hucker: $\mathrm{n}^{\circ}$ 3564.4763. Charge militaire de l'époque des Tang et des Song. 
en l'occurrence le roi du Grand Soleil (Vairocana) ${ }^{152}$. Paek Sŭnghyŏn trouva ensuite crédit auprès de puissants, tels que Kim Chun (?-1268) ${ }^{153}$, sous le règne de Wŏnjong (r. 1259-1274), mais apparemment sans être nommé au bureau de la Divination.

À partir du règne de Kongmin et jusqu'à la fin de la dynastie, la question du transfert de la capitale, en tant que mesure nécessaire pour susciter un «nouvel essor» chunghŭng, refit surface ${ }^{154}$ et mobilisa les devins d'État. D'un point de vue technique, celle-ci prit fréquemment la forme de tirages au sort effectués par les devins ou conseillers d'État dans le Temple des ancêtres de la dynastie, ou bien dans le pavillon funéraire du fondateur T'aejo. Il semble qu'alors la théorie des Trois So ne faisait plus recette, de plus en plus contestée par une partie de la haute administration.

C'est dans ce contexte, et parmi les mentions diaphanes relatives aux astrologues de la cour, que, par contraste, s'impose la figure honnie de Sin Ton $(?-1371 \text { ? })^{155}$. Le personnage, fils d'une esclave de monastère ${ }^{156}$, moine collecteur itinérant ${ }^{157}$, illettré, connut une ascension fulgurante à partir de $1364^{158}$. Kongmin le prit pour maître et lui octroya, en 1365 , un titre de noblesse ainsi que les plus

152. Sur les dires de Paek Sŭnghyŏn fut construit le monastère de Hyŏlgu, en tant que lieu de séjour permanent de Vairocana (以爲大日王常住處). KRS: 123,6b.

153. 金俊 (金仁俊). Un des militaires qui renversa le régime des Ch'oe d'Ubong en assassinant Ch'oe Ǔi (崔誼,?-1258) en 1258 avec la complicité de Yu Kyŏng (柳璥, 1211-1289) et Pak Songbi (朴松庇,?-1287). De basse extraction, il parvint, grâce à son mérite militaire, à monter dans la carrière sous les Ch'oe. Il devint sujet méritant de deuxième classe pour avoir contribué à la restauration du pouvoir royal des Wang.

154. En 1356, 1360, 1382, 1390, 1391.

155. Il fait l'objet d'une longue biographie dans la catégorie des «Rebelles». KRS : 132. Les historiographes le dépeignent comme un personnage dépravé, cruel, buveur, libidineux et démagogue. Selon le KRS, Sin Ton aurait eu un fils d'une esclave, qui aurait été intronisé en tant que fils de Kongmin en 1374, le roi U (r. 1374-1388). On trouve une traduction annotée de la Biographie de Sin Ton en Bruneton 2002: 434-462.

156. Selon le KRSCY, il s'agirait du monastère Okch’ŏn de Yŏngsan (靈山縣 玉泉寺; KRSCY : 28,11a), alors que le KRS mention le monastère Okch’ŏn du district de Kyesŏng (桂城縣 玉川寺; KRS : 132,1a).

157. Son nom de moine était P'yŏnjo 遍照 alias Sin Ton du clan des Sin de Yŏngsan 靈山 辛氏. La description du KRS suggère qu'il n'était pas un religieux ordonné, mais qu'il vivait d'extorsion des fidèles sous prétexte de collecte de dons. Il utilisa définitivement son nom de laïc à partir de 1365.

158. KRSCY : 28,11a (1365 ?). Le KRS donne la date de Kongmin 13: 1364. Introduit à la cour par Kim Wŏnmyŏng (金元命,?-1370), le souverain reconnut dans les traits du moine, un religieux qui, au cours d'un rêve de la nuit précédente, l'avait sauvé providentiellement. Il se fit pousser les cheveux et se donna l'apparence d'un ascète (dhuta). Le roi lui donna le titre de Ch’ŏnghan kŏsa 靜閑居士. 
hautes fonctions, le propulsant à la tête du gouvernement. Outre la présidence des conseils, il occupa la direction du bureau des Affaires bouddhiques en cumul avec celle du bureau de la Divination. Le caractère cumulé de la fonction est instructif en soi de la nature étroite des relations entre les deux bureaux. Cependant, la description de ses activitiés dans les matières divinatoires passe le plus souvent au second plan de celles des réformes institutionnelles qu'il entreprit ${ }^{159}$. Sin Ton, en tant chef des devins, ne fit pas preuve d'originalité : il se référa à la littérature prophétique. Il répondit favorablement à la sollicitation d'un moine du grand monastère de Yŏnbok de la capitale pour aménager le monastère conformément aux anciennes prophéties, montrant son intérêt pour la géomancie des édifices bouddhiques. À partir de 1366, Sin Ton recommanda au roi de faire donner des cérémonies bouddhiques nouvelles: les assemblées de Munsu (Manjusri), pour lui assurer une descendance qui n'arrivait pas. Il conçut surtout, en 1367, l'ambitieux projet de déplacer la capitale en se référant aux écrits secrets de Tosŏn. Il se rendit pour cela successivement dans la capitale de l'Ouest, puis à Ch'ungju en 1369. Les deux tentatives n'aboutirent à aucun résultat. Finalement, au nom de la théorie des Trois So, il fit édifier un palais secondaire dans les deux villes. Avant d'être condamné à mort pour avoir fomenté un complot contre le roi en 1371, le pseudo moine fut l'objet de critiques virulentes de la part des hauts fonctionnaires et du clergé monastique régulier. Dans le milieu des devins, s'organisa une forme de résistance contre Sin Ton. Le président du bureau d'Astrologie, Chin Yŏngsŏ (?- ?), quant à lui, s'arrangea pour faire échouer les projets de déplacements du souverain en persuadant Kongmin de leur caractère néfaste.

\section{L'école de Tosŏn et la question de la transmission des savoirs}

De tout ce qui précède, il ressort avec une certaine évidence qu'en dépit de l'influence incontestable de canons de la divination chinoise des Tang et des Song ${ }^{160}$, la cour du Koryŏ, dans la continuité probable des pratiques spéculatives

159. Les historiographes suggèrent que le souverain se servit d'un personnage sans attaches à la noblesse et aux intérêts des coteries pour procéder aux épurations souhaitées par le roi dans l'administration et dans les rangs des sujets méritants qui s'étaient distingués dans la lutte contre les invasions des Turbans Rouges (1359-1361). Sin Ton écarta nombre de hauts fonctionnaires pour placer ses partisans. Il accapara le pouvoir avec le titre de «conseiller»ch'ŏmŭi 僉議 et traita le roi d'égal à égal.

160. En plus des ouvrages, la cour du Koryŏ recourut parfois à l'emploi de transfuges des Song: ce fut le cas de Chang Wan (張琬,?-?), reçu en 1057 à la cour de Kaesŏng. Spécialiste de la pratique de la divination par le «cycle caché et les esprits des troncs célestes (trois $k i$ et six im 遁甲三奇六壬) ». Il fut nommé «préposé à la température» 
caractéristiques de la période troublée précédant l'instauration de la dynastie (898-935), élabora une culture divinatoire propre. Il est vraisemblable que celle-ci prit pour point de départ des enjeux politiques aussi fondamentaux que la légitimation de la politique du fondateur vis-à-vis du choix des nouvelles capitales, principale (Kaegyŏng) et secondaire (P'yŏngyang), ainsi que le soutien à la construction des édifices bouddhiques. La fonction légitimante de telles théories explique l'officialisation progressive de la figure du moine Tosŏn, associée au mythe de fondation de la dynastie ${ }^{161}$, et promue par étapes au cours de la période (jusqu'à recevoir le titre inédit de «maître national de géomancie » chiri kuksa en 1308) ${ }^{162}$, mais aussi et surtout à la formation d'une véritable école divinatoire traitant principalement de sujets liés à la géomancie. Au cours d'un tel processus, il est instructif de constater que Tosŏn fut affilié symboliquement au maître de dhyâna Yixing des Tang, parce que la tradition attribuait à ce dernier la rédaction d'une partie des traités de référence servant à former les devins du Koryŏ, notamment par le biais de la section de géoscopie ${ }^{163}$. On peut affirmer que la mise en exergue de l'école de Tosŏn est une des grandes leçons du Koryŏsa sur la divination d'État. C'est en tout cas mon hypothèse, bien qu'en Corée du Sud, les théories de Tosŏn ne soient pas présentées en tant qu' «école». À l'appui indirect d'une telle thèse, on observe dans la durée l'existence de résistances internes à ces théories par certains devins et astrologues, suggérant que la divination officielle du Koryŏ était influencée par différents courants, reflet de sa vitalité. Toutefois, il n'est pas possible d'identifier les écoles concurrentes. Au cours de la période, le fait que les zélateurs des théories de l'école de Tosŏn furent généralement intégrés dans l'administration comme membres des bureaux spécialisés, est la preuve la plus solide de son caractère institué ${ }^{164}$.

Il est vraisemblable que l'école de Tosŏn s'enrichit au cours du temps, sinon de nouveaux textes, du moins de gloses qui s'amalgamèrent à des noyaux

(太史監候, de $9^{\mathrm{e}}$ échelon inférieur) après avoir été testé sur ses connaissances (KRS : 8,6a-b).

161. KRS: Segye (世系, citations d'ouvrages du XII et du XIII ${ }^{\mathrm{e}}$ siècles).

162. 地理國師. $C f$. Promotion en quatre étapes. Bruneton 2002: 812.

163. La filiation de Tosŏn à Yixing relevait vraisemblablement d'une volonté politique de légitimer les théories du moine du Silla et de leur conférer du prestige en les associant à la grande tradition divinatoire des Tang (Bruneton 2012).

164. Le cas de Kim Wije est exemplaire à cet égard: sa connaissance des théories de Tosŏn lui valut d'être employé comme expert dans le conseil temporaire pour la construction d'un palais secondaire dans la capitale de l'Ouest en 1106 (KRSCY : 7,17a; KRSCY: 96,39b). Il est même possible qu'il participât, associé à Mun Sang, au conseil temporaire pour la fondation de la capitale du Sud en 1101, ainsi qu'à la compilation du Haedong pirok en 1106. 
originaux. On ignore toutefois l'impact de mesures d'épuration de la littérature divinatoire comme celle qui intervint en 1106 ( $c f$. supra). Les documents étaient conservés dans les fonds des archives des bureaux d'Astrologie et de Divination, comme le confirment a posteriori les Annales de règne du Chosŏn $\mathrm{au} \mathrm{XV}^{\mathrm{e}}$ siècle. On peut penser qu'en raison du caractère politique sensible de ces textes, leur accès était limité aux spécialistes, aux hauts fonctionnaires, et à ceux que ces derniers autorisaient. Dès lors, considérant la relative diversité du statut des adeptes (opportunistes ou convaincus) de l'école de Tosŏn, il est possible que ceux-ci aient constitué une sorte de réseau au sein duquel étaient transmis textes et interprétations. On constate en effet, à la lecture du Koryŏsa, que l'activité des devins d'État se caractérisait implicitement par sa nature collégiale : pour communiquer avec les politiques, ceux-ci se devaient de parler à l'unisson. Autrement dit, la somme des compétences mises en œuvre, réparties hiérarchiquement entre les différents membres, devait aboutir à une formulation unique portée par le responsable du bureau. Cela explique en partie le caractère anonyme des rapports du bureau de la Divination mentionnés dans l'histoire officielle. La persistance de l'influence de l'école de Tosŏn entre le milieu du $\mathrm{XI}^{\mathrm{e}}$ siècle et jusqu'à la fin $\mathrm{XIV}^{\mathrm{e}}$ siècle pose donc de manière aiguë et implicite la question de la transmission des savoirs en matière de divination.

\section{Les modes de transmission des théories de Tosŏn}

L'histoire officielle est avare de données à propos des modes de transmission des connaissances, à plus forte raison des techniques et savoir-faire comme ceux des arts divinatoires, considérés comme subalternes. Dans le cas des théories de Tosŏn, il n'est pas difficile de supposer que les textes étaient, sinon enseignés, tout au moins consultés et étudiés (sinon copiés) ${ }^{165}$ par les devins fonctionnaires, puisque conservés - nous l'avons dit - dans les locaux des bureau spécialisés. À ce premier cercle d'initiés put se joindre, en certaines circonstances, un deuxième cercle de fonctionnaires, non officiellement devins, mais qui entretenaient relations et affinités avec le milieu des devins de la cour. À cet égard, il est légitime d'envisager deux types de relations privilégiées : fondées sur l'hérédité ou sur la relation de maître à disciple. En effet, l'apprentissage des

165. Le KRS atteste la pratique de la conservation de textes chez les fonctionnaires kajang (家藏). Le cas le plus célèbre est celui de Ch'oe Anje (崔齊顔,?-1046) chez qui, à sa mort, l'on retrouve une version du testament politique du fondateur (KRSCY : 4,38a). On peut supposer que l'entourage immédiat des devins fonctionnaires pouvait donc avoir accès à la littérature spécialisée. Ce point rejoint la question de la transmission par l'hérédité. L'interdiction promulguée en 1417 de la détention à titre privé de textes divinatoires jugés non conformes, en est la preuve indirecte (CWS : 1417.12.15.). 
arts divinatoires commençait, semble-t-il, au sein des familles ${ }^{166}$ : O Yunbu, devin modèle du Koryŏsa (cf. supra), était issu d'un tel clan (les O de Puhŭng) ${ }^{167}$. Son gendre, Kang Mi (?-?) et le père de celui-ci, Kang Hŏn (?-?), impliqués dans l'affaire de la divination du site de la tombe du roi Ch'ungnyŏl en $1309^{168}$, étaient sans doute, eux aussi, issus d'un clan de devins héréditaires ${ }^{169}$. Un autre exemple de ce type de transmission est donné par Yŏng Ŭ $i^{170}$, dont le père, Yŏng Sang (??), avait été directeur du bureau d'Astrologie ${ }^{171}$. Concernant la relation de maître à disciple, l'exemple le plus explicite est celui de Myoch'ŏng du monastère de Chunghŭng et de Paek Suhan, de l'école de la capitale de l'Ouest. Un passage instructif de la Biographie de Myoch'ŏng, par sa rareté et son originalité, nous renseigne même sur le caractère ésotérique de la transmission de techniques assimilées au Cycle caché ${ }^{172}$. Le cas de l'association entre le devin fonctionnaire Mun Sang et celui de Kim Wije est moins explicite et est difficile à interpréter à cause de notre ignorance des antécédents des deux personnages.

Nous avons présenté jusqu'ici les données les plus remarquables relatives aux devins qui intervinrent dans l'histoire du Koryŏ en tant qu'acteurs influents à

166. Parmi les quelques dizaines de noms de devins et d'astrologues qui nous sont parvenues (87 selon le recensement de Kim Ch'anghyŏn), on trouve plusieurs mentions des clans Mun (Mun Sang, Mun Chang'yu, Mun Sŏyŏng), Ch'oe (Ch'oe Sagyŏm, Ch'oe Chaho, Ch'oe Chahyŏn), Sŏ (Sŏ Ung, Sŏ P'il, Sŏ Hŭi), Yu (Yu P'aeng, Yu Tŭkso) et Yang (Yang Wŏnho, Yang Kwan'gong, Yang Nin, Yang Hae). Le problème réside dans l'identification des lieux d'origine patronymiques pon'gwan 本貫 et la connaissance des biographies des intéressés. Le nombre de pon'gwan connus des membres des bureaux de la Divination et de l'Astrologie serait de l'ordre de 18,4\%. Pour Kim Ch'anghyŏn, il s'agit de «patronymes de terroir» t'osŏng 土姓, suggérant qu'ils étaient issus de clans locaux, avec peu d'ancêtres prestigieux (Kim 1992: 137).

167. 復興吳氏. KRS : 122,5b : 世爲太史局官. O Yunbu est l'unique devin connu issu de ce clan.

168. KRS : 33,19b. À l'occasion d'une visite rituelle du roi Ch'ungsŏn à la tombe de son père (le roi Ch'ungnyŏl), le site de la tombe Ko (高陵) fut soupçonné de ne pas être faste. Les membres du conseil qui effectuèrent le choix du site furent emprisonnés ainsi que deux des petits-fils d'O Yunbu.

169. Kim 1992: 145.

170. $C f$. supra p. 65 .

171. 司天監 榮尙. 監 $k a m, 3^{e}$ échelon inférieur. Il fut envoyé en exil dans une île. KRS : $123,2 \mathrm{a}$.

172. À l'occasion de la pratique de la technique dite de la «Marche de la Tente de jade du Grand Un», exécutée au moment de l'ouverture des travaux pour la reconstruction du palais royal à Kaesŏng en 1132, les historiographes prêtent à Myoch’ŏng d'avoir eu la pensée de transmettre la technique à Paek Suhan, lorsqu'il serait vieux, afin qu'elle ne fût pas connue d'un grand nombre de personnes. Il en aurait reçu l'enseignement d'un dénommé Kang Chŏnghwa (康靖和, inconnu) instruit par Tosŏn (KRS : 127, 30a). 
la cour, justifiant leur consignation par les chroniqueurs. Le Koryŏsa mentionne également, mais de façon encore plus discrète, l'existence de devins œuvrant parmi la population parce qu'étant eux-mêmes généralement issus du peuple.

\section{Les praticiens de la divination domestique et privée}

Au Koryŏ, les devins et astrologues pratiquant au sein de la population ${ }^{173}$ dans un cadre domestique et privé constituent la catégorie à propos de laquelle nous disposons du moins d'informations. On sait toutefois qu'il existait de multiples interdits formulés parfois en termes d' «évitements du yin et du yang» ŭmyang kugi ${ }^{174}$. Quand elles existent, les données sont le plus souvent indirectes, témoignant par exemple d'une étape dans le parcours de praticiens ayant connu une promotion sociale, comme ce fut le cas des anciens moines Chu Yŏnji (cf. supra) ou Cho Sin'gyŏng (?-?; XIV ${ }^{\mathrm{e}}$ siècle) ${ }^{175}$, ou bien de leur caractère extraordinaire (au sens littéral et généralement négatif). Dans les milieux monastiques, artisans et experts en géomancie exerçaient localement dans le cadre de procédures de construction d'édifices bouddhiques, et bénéficiaient d'une relative liberté de déplacement, mais ils sont absents de l'histoire officielle. Enfin, il ne faudrait pas oublier les lettrés retirés, fonctionnaires subalternes ou moines, qui, par goût personnel de l'étude, mirent en pratique leurs connaissances. Dans le Koryŏsa, il est possible de mettre en évidence deux catégories de devins privés: les moines dissidents et les aveugles.

\section{Moines dissidents}

Les moines devins formaient au Koryŏ une des catégories de spécialistes parmi les plus actives auprès de la population. En raison des règles de compilation de l'histoire dynastique, leur mention ne se justifiait toutefois pas a priori dans le Koryŏsa. On trouve cependant la citation ponctuelle et laconique de cas limites consignés pour avoir troublé l'ordre public et enfreint le domaine d'activités réservé aux devins fonctionnaires. Ainsi, pour l'année 1101, sont cités deux moines considérés comme subversifs. Dans la capitale, le moine Kwanggi (?-?) rattaché au monastère de Kwangmyŏng, avec deux complices fonctionnaires subalternes, falsifia la rédaction d'un ouvrage sur le yin-yang $\breve{m}$ yyang s $\breve{o}^{176}$. L'affaire fut révélée et l'intéressé fut passible de bastonnade et d'exil. Au cours

173. La classe du bon peuple yangmin 良民 était inférieure à celle des clercs de province hyangni 鄉吏 et des lettrés fonctionnaires sadaebu 士大夫.

174. 陰陽拘忌.

175. KRS: $124,24 b$. 曹莘卿. Bruneton 2002 : 414.419.

176. 光器. KRS: $11,27 a$. 
de la lunaison suivante, à P'yŏngju (actuel P'yŏngsan, prov. Hwanghae), un moine maléfique yosŭng, Kakchin (?-?), aurait, quant à lui, tenu des propos erronés sur le yin et le yang, et abusé les foules. Il fut envoyé en exil à Kokchu (actuel Koksan, prov. Hwanghae) ${ }^{177}$. Ici encore, nous restons dans l'ignorance $\mathrm{du}$ fond de l'affaire. On remarque par ailleurs que le début du XII siècle connut une certaine effervescence dans le domaine des affaires divinatoires, possiblement causée par la difficulté de contrôler le foisonnement des théories et leur diffusion parmi la population par l'intermédiaire des religieux. En cette même année 1101, la cour s'apprêtait en effet à faire le choix d'un site pour la nouvelle capitale secondaire du Sud. Dans le même temps, il était question d'interdire des rassemblements de religieux et de membres du peuple formant les assemblées dites des Dix mille Bouddha manbul hoe, et tenant des «discours extravagants ${ }^{178}$. On peut douter de l'efficacité de l'interdiction : trente ans plus tard, c'est au tour des «groupes d'encens (fidèles bouddhistes) des Dix mille Bouddhas » manbul hyangdo, de faire l'objet d'une interdiction par le «Conseil du yin et du yang» ümyang hoeso ${ }^{179}$.

\section{Devins aveugles}

À l'époque du Koryŏ, la divination, en raison de son caractère lucratif, semble avoir été une pratique permettant d'assurer la subsistance de certaines catégories de population : aveugles, laïcs et religieux. On trouve ainsi plusieurs mentions de devins aveugles dans l'histoire officielle : les mae pok maeng' $i{ }^{180}$. Ils étaient assimilés au vil peuple et n'avaient pas le droit de bénéficier d'allocations foncières à l'instar des esclaves (d'État ou privés), des artisans et des marchands ${ }^{181}$. En dehors de la divination (tirage de pronostics), les aveugles étaient connus pour leur activité d'incantations bouddhiques ${ }^{182}$. Le Koryŏsa rapporte nominalement l'activité de deux devins aveugles, impliqués dans des affaires politiques aux $\mathrm{XIII}^{\mathrm{e}}$ et $\mathrm{XIV}^{\mathrm{e}}$ siècles, qui ne se terminèrent pas à leur avantage. Le premier, le moine aveugle Paengnyang (?- ?), fut employé pour

177. 妖僧 覺眞. KRS: $11,27 \mathrm{~b}$.

178. 萬佛會. KRSCY: 6,39b.

179. 萬佛香徒. KRS : 85,11a (陰陽會所).

180。賣卜盲人。

181. KRS : $78,41 \mathrm{~b}$.

182. CWS : $1394.1 .16 ; 1394.4 .17 ; 1405.1 .11 ; 1417.9 .2 ; 1418.2 .11$. Au Chosŏn, on note la pratique de faire prier des aveugles au monastère de Myŏngt'ong 明通寺. Voir aussi Ko Sang'an 高尚顔, 泰村集 卷4, 叢話; Pak Tongnyang 朴東亮, 寄齋雜記, 歷朝舊 聞; Cho Kyŏngnam 趙慶南, 續雜錄, 戊辰; Yi Kyugyŏng 李圭景, 五洲衍文長箋散 稿, 經史篇, 論史類, 明通寺辨證說. 
juger du caractère faste de la peine infligée à plusieurs fonctionnaires qui avaient fomenté un complot contre Kim Chun en 1258, peu de temps avant que ce dernier élimine le dernier rejeton du clan des Ch'oe d'Ubong. Il finit par faire jeter à la mer les comploteurs, et réduire leur maisonnée en esclavage ${ }^{183}$. Le second devin aveugle, Sŏk Ch'ŏllok (?-?), est cité dans la biographie d'O Int'aek (?-?; $\mathrm{XIV}^{\mathrm{e}} \mathrm{s}$.) ${ }^{184}$, car lorsque ce dernier, en 1364, fut envoyé en exil (à Ch'ŏngp'ung) pour corruption, les membres de sa famille proche eurent recours aux services de l'augure pour savoir quand ceux qui l'avaient condamné (Ch'oe Yŏng, Yi Kwisu) seraient eux-mêmes bannis de la cour. La réponse de Sŏk Ch'ŏllok fut ébruitée, et valut aux protagonistes de l'affaire d'être punis: le devin fut exilé avec plusieurs membres du clan des $\mathrm{O}$, et reçut la bastonnade ${ }^{185}$. Après la chute de la dynastie des Wang, les annalistes du début du Chosŏn continuèrent de mentionner des devins aveugles, révélant une continuité dans l'organisation et les pratiques sociales du Koryŏ ${ }^{186}$.

\section{Conclusion}

En dépit des limites inhérentes aux sources, toute recherche sur la divination à l'époque du Koryŏ ne saurait faire l'économie d'une investigation minutieuse de l'histoire officielle, principal apport d'informations sur le sujet. En ce sens, la tentative de synthèse ici présentée constitue un premier effort d'analyse du phénomène politique, technique, intellectuel et social complexe que représentent la divination et ses acteurs. Ce travail contribue d'abord à caractériser la culture divinatoire de la période étudiée: tout en bénéficiant des apports théoriques fondamentaux des Tang et des Song, celle-ci se trouvait placée au cœur du système politique et ritualiste du Koryŏ. Pour cette raison, le bouddhisme, en tant que religion d'État de la dynastie des Wang, participait, par ses enseignements, ses monastères, ses rituels, et les membres de son clergé, pour une part importante aux activités divinatoires. La collaboration nécessaire entre devins séculiers et religieux en vue de remplir leur mission commune de protection de la dynastie, était facilitée par l'usage des structures souples et collégiales qu'étaient les conseils provisoires, mais aussi par un système - au moins ponctuel - de cumul de fonctions entre les bureaux de la Divination et celui des Affaires bouddhiques.

183. KRS : 130,14a. 以盲僧伯良卜其吉凶.

184. 石天祿. KRS: 114,19b-21a. 吳仁澤 (?-?). Sujet méritant qui s'était distingué dans la lutte contre les Turbans Rouges et la récuparation de Kaesŏng après son invasion. Il reçut les faveurs du roi Kongmin.

185. KRS : 114,20b-21a. KRSCY : 28,7b-8a.

186. Mentions avant le règne de Sejong (世宗, r. 1419-1450) : CWS : 1394.1.6; 1394.2.6; $1394.2 .25 ; 1394.4 .17 ; 1399.6 .1 ; 1403.6 .29 ; 1405.1 .11 ; 1417.9 .2 ; 1417.9 .21 ; 1418.2 .11$. 
Outre l'activité de moines devins qualifiés de sulsŭng à la cour à partir du XII ${ }^{\mathrm{e}}$ siècle, le Koryŏsa met en évidence l'existence d'une école de divination que nous avons appelée ici «école de Tosŏn», fonctionnant comme une idéologie de référence dans le milieu des devins fonctionnaires entre le $\mathrm{XI}^{\mathrm{e}}$ siècle et la fin de la dynastie. Centrée sur les questions de choix de sites fastes pour le séjour du souverain, elle produisit le phénomène original de la géomancie des capitales et de la géomancie «de soutien» par les édifices bouddhiques. Sans faire nécessairement l'unanimité parmi les experts, elle constitua une source d'inspiration constante et proposa des solutions de réorganisation du territoire dans les situations de crise. En tant qu'élément de légitimation du pouvoir de la dynastie des Wang, elle était inattaquable auprès des souverains, mais dut néanmoins renforcer son prestige (sinon ses théories) par l'affiliation de Tosŏn du Silla à Yixing, révélant par là une certaine parenté avec la culture divinatoire des Tang, notamment du point de vue de ses relations avec le bouddhisme.

Notre étude montre que l'école de Tosŏn eut de nombreux adeptes, séculiers ou religieux, et que leur action politique ne fut viable que par l'existence de réseaux de connivence de différents membres de l'État, à commencer par les devins eux-mêmes. Ainsi, l'école de Tosŏn renvoie à la problématique plus générale de la transmission des connaissances divinatoires au Koryŏ. Celle-ci est délicate à traiter en raison non seulement de l'état des sources, mais aussi parce que la catégorie sociale des devins fonctionnaires, se définissant avant tout par la reconnaissance de compétences plus que par la naissance, remet en question imperméabilité supposée des grandes distinctions administratives et sociales (en particulier entre séculiers et religieux) et bouleverse les cadres rigides posés par l'historiographie. Il conviendrait de compléter la présente contribution sur la base des travaux de Kim Ch'anghyŏn, qui, à ma connaissance, est le premier chercheur à avoir établi un recensement nominal quasi exhaustif (87) des devins et astrologues d'État dans les sources du Koryŏ, et à avoir reconstitué partiellement leurs origines sociales et le déroulement de leurs carrières. 


\section{Bibliographie}

\section{Sources primaires}

Hong, Ponghan 洪鳳漢 (1983). Tongguk munhŏn pigo 東國文獻備考. Séoul, Myŏngmundang 明文堂.

No, Sasin 盧思愼 et al. (1994). Tongguk yŏji sŭngnam 東國輿地勝覽. Séoul, Myŏngmundang 明文堂.

Sŏ, Kŏchŏng et al. 徐居正 (1994). Tongmunsŏn 東文選. Séoul, Minjok Munhwa kanhaenghoe 民族文化刊行會.

HAN'GUK KWAHAKSA HAK'HOE 韓國科學史學會 編 (1982). Sŏun'gwan chi 書雲觀志. Séoul, Sŏngsin yŏja taehakkyo ch'ulp'anbu 誠信女子大學校 出版部.

KIM, Kyŏnmyŏng 金見明, Iryŏn一然, Ch'oe Namsŏn 崔南善 編 (1997). Samguk yusa 三國遺事. Séoul, Sŏmun Munhwasa 瑞文文化社.

CHǑNG, Inji 鄭麟趾 et al. (1983). Koryŏsa 高麗史. Séoul, Asea Munhwasa 亞細亞文 化社.

KIM, Chongsŏ 金宗瑞 et al. (1973). Koryŏsa chŏryo 高麗史節要. Séoul, Asea Munhwasa 亞細亞文化社.

Xu, Jing 徐兢 (1972). Gaoli tujing 高麗圖經. Séoul, Asea Munhwasa 亞細亞文化社.

Hŏ, Hŭngsik 허흥식 (1984). Han'guk kŭmsŏk chŏnmun 韓國金石全文. Séoul, Asea Munhwasa 亞細亞文化社.

\section{Webographie (sources primaires)}

gsm.nricp.go.kr (National Research Institute of Cultural Heritage)

sillok.history. go.kr (The Annals of the Choson Dynasty)

db.itkc.co.kr (DB of Korean classics)

ebti.dongguk.ac.kr (Hanguk Bulgyo Chonso Retrieval System)

www.cbeta.org (Chinese Buddhist Electronic Text Association, CBETA)

\section{Sources secondaires}

En langues non occidentales

KIM, Ch'anghyŏn 金昌賢 (1992). Koryŏ sidae ilgwan-e kwanhan ilgoch'al - ilgwan-ŭi yŏk'hal-gwa kŭ chiwi-rŭl chungsim-ŭro 高麗時代 日官에 관한 一考察 - 日 官의 役割과 그 地位를 중심으로 -. Séoul, Sahak yŏn'gu 史學研究 no 45 (1992.8) : 77-153.

KIM, Ilgwŏn 김일 권 (2002.6). Ch'ŏnjisŏsangji-ŭi yŏksajŏg-wa saryojŏk kach’i 天地瑞 祥誌의 역사적와 사료적 가치. Séoul, Han'guk kodaesa yŏn'gu 韓國古代史研 究 $n^{\circ} 26: 221-268$.

KIM,Ilgwŏn 김일권 (2011). Koryŏsa chayŏnhak-kwa ohaengji yŏkchu 高麗 史 자연학과 오행지 역주. Sŏngnam (Kyŏnggido), Han'gukhak chung'ang yŏn'guwŏn ch'ulp'anbu 韓國, 學中央研究院出版部.

KIM, Pyŏnggi 金炳機 (1982). Han'guk kwahaksa 韓國科學史. Séoul, Iu ch'ulp'ansa 二友출판사.

KIM, Yong'un 金容雲, KIM Yongguk 金容局 (1977). Han'guk suhaksa 韓國數學史. Séoul, Yŏrhwadang 悅話堂. 
HAN, Kimun 韓基汶 (1998). Koryŏ sawŏn-ŭi kujo-wa kinŭng 高麗寺院의 構造와 機 能. Séoul, Minjoksa 民族社.

Hŏ, Hŭnsik 許興植 (1993). Koryŏ pulgyosa y'ŏn'gu 高麗佛呚史手研究. Séoul, Ilchogak 一潮閣.

Hŏ, Hŭnsik 許興植 (1994). Han'gug-ŭi komunsŏ 한국의 古文書. Séoul, Minŭmsa 民 音社.

PYŎN, T'aesŏp 邊太雍 (1982). Koryŏsa-ŭi yŏn'gu 高麗史의 研究. Séoul, Samyŏngsa 三英社.

Sŏ, Yun'gil 徐閏吉 (1993). Koryŏ sidae-ŭi milgyo 高麗密敎思想史研究. Séoul, Pulgwang ch'ulp'anbu 불광출판부.

YI, Hŭidŏk 李熙德 (1984). Koryŏ yugyo chŏngch'i sasang-ŭi yŏn'gu 高麗儒敎 政治 思想의 研究. Séoul, Ilchogak 一潮閣.

YI, Pyŏngdo 李丙壽 (1980). Koryŏ sidae-ŭi yŏn'gu 高麗時代의 研究. Séoul, Asea Munhwasa 亞細亞文化社.

En langues occidentales

BRunEton, Yannick (1997). «Séoul à l'époque Koryŏ - Capitale du Sud sous la dynastie des Wang ». Revue de Corée, $\mathrm{n}^{\circ} 101$. Séoul, Commission Nationale coréenne pour l'Unesco: 230-260.

BRUNETON, Yannick (2002). Les moines géomanciens de Koryŏ - une étude critique des sources. Thèse de doctorat. Paris, Université Paris Diderot.

BRUNETON, Yannick (à paraître). «Le bouddhisme ésotérique milgyo dans la Corée médiévale: entre école bouddhique, lignée spirituelle et école de pensée, hypothèse d'une «école coréenne de Yixing» ( $\mathrm{IX}^{\mathrm{e}}-\mathrm{XV}^{\mathrm{e}}$ siècles). Louvain, Peeters.

Kalinowski, Marc (1991). Cosmologie et divination dans la Chine ancienne - Le Compendium des Cinq Agents (Wuwing dayi, $\mathrm{vI}^{\mathrm{e}}$ siècle). Paris, EFEO.

Sørensen, Henrik (2006). «Esoteric Buddhism under the Koryŏ in the Light of the Greater East Asian Tradition ». International Journal of Bouddhist Thought and Culture ${ }^{\circ} 7$ : 55-94.

Sørensen, Henrik (2010). «Worshipping the Cosmos: Tejaprabha Rituals under the Koryŏ ». International Journal of Bouddhist Thought and Culture vol. 15 : 1-21.

La traduction des charges administratives provient du Répertoire Historique de l'Administration coréenne (1890.1986) de Maurice Courant; en cas d'absence dans le Répertoire, elle se fonde sur les explications fournies par A Dictionary of Official Titles in Imperial China de Hucker, Charles O.

$\begin{array}{ll}\text { Abréviations } \\ \text { CWS } & \text { Chosŏn wangjo sillok } \\ \text { HKC } & \text { Han'guk kŭmsŏk chŏnmun } \\ \text { KRS } & \text { Koryŏsa } \\ \text { KRSCY } & \text { Koryŏsa chŏryo } \\ \text { TYS } & \text { Tongguk Yŏji sŭngnam }\end{array}$


